\title{
On local packings of the cross-polytope
}

\author{
Ji Hoon Chun* \\ Institut für Mathematik \\ Technische Universität Berlin \\ Berlin, Germany \\ chun@math.tu-berlin.de
}

Submitted: Sep 4, 2019; Accepted: May 18, 2020; Published: Aug 21, 2020

(c) The author. Released under the CC BY-ND license (International 4.0).

\begin{abstract}
The problem of finding the largest number of points in the unit cross-polytope such that the $l_{1}$-distance between any two distinct points is at least $2 r$ is investigated for $r \in\left(1-\frac{1}{n}, 1\right]$ in dimensions $n \geqslant 2$ and for $r \in\left(\frac{1}{2}, 1\right]$ in dimension 3. For the $n$-dimensional cross-polytope, $2 n$ points can be placed when $r \in\left(1-\frac{1}{n}, 1\right]$. For the three-dimensional cross-polytope, 10 and 12 points can be placed if and only if $r \in\left(\frac{3}{5}, \frac{2}{3}\right]$ and $r \in\left(\frac{4}{7}, \frac{3}{5}\right]$ respectively, and no more than 14 points can be placed when $r \in\left(\frac{1}{2}, \frac{4}{7}\right]$. Also, constructive arrangements of points that attain the upper bounds of $2 n, 10$, and 12 are provided, as well as 13 points for dimension 3 when $r \in\left(\frac{1}{2}, \frac{6}{11}\right]$.
\end{abstract}

Mathematics Subject Classifications: 05B40, 52C17

\section{Introduction}

Let $K$ and $L$ be origin-symmetric convex sets in $\mathbb{R}^{n}$ with nonempty interiors. A set $D \subset \mathbb{R}^{n}$ is a (translative) packing set for $K$ if, for all distinct $\mathbf{x}, \mathbf{y} \in D$,

$$
(\mathbf{x}+\operatorname{int}(K)) \cap(\mathbf{y}+\operatorname{int}(K))=\varnothing,
$$

where int $(K)$ is the interior of $K$. For $r>0$, we consider the problem of finding the maximum number of points in a packing set $D$ of $r K$ that is contained in $L$. This quantity will be denoted by

$$
\gamma(L, K, r):=\max \left\{|D| \mid D \subset L \text { and }\|\mathbf{x}-\mathbf{y}\|_{K} \geqslant 2 r \text { for any } \mathbf{x}, \mathbf{y} \in D, \mathbf{x} \neq \mathbf{y}\right\},
$$

*Supported by the Deutsche Forschungsgemeinschaft (DFG) Graduiertenkolleg "Facets of Complexity/Facetten der Komplexität" (GRK 2434). 
where $\|\mathbf{x}\|_{K}=\min \{\lambda \mid \lambda \geqslant 0$ and $\mathbf{x} \in \lambda K\}$ and for a set $S$, its cardinality is denoted by $|S|$. If $K=L$ then we use the notation $\gamma(K, r)$ as a shorthand for $\gamma(L, K, r)$. We will only deal with the situation where both $K$ and $L$ are the unit cross-polytope $C_{n}^{*}=\left\{\mathbf{x} \in \mathbb{R}^{n}\left|\sum_{i=1}^{n}\right| x_{i} \mid \leqslant 1\right\}$.

A set $D$ in $C_{n}^{*}$ with $k$ points such that the $l_{1}$-distance between any two distinct points is greater than or equal to $2 r$ is equivalent to a packing set for $r C_{n}^{*}$ such that each set $\mathbf{x}=r C_{n}^{*}, \mathbf{x} \in D$, is contained inside the set $(1+r) C_{n}^{*}$. Unless otherwise specified, we will use "distance" to mean the $l_{1}$-distance. The vertices of $C_{n}^{*}$ are the $2 n$ unit vectors $\left\{ \pm \mathbf{e}_{i} \mid i \in\{1, \ldots, n\}\right\}$, so the distance between any two distinct vertices is 2 , which implies that $\gamma\left(C_{n}^{*}, r\right)=1$ for any $r>1$ and $\gamma\left(C_{n}^{*}, r\right) \geqslant 2 n$ for $r \leqslant 1$.

The case $r=\frac{1}{2}$ is related to the topic of kissing numbers. Let $K \subset \mathbb{R}^{n}$ be a convex body, that is, both compact and convex. The (translative) kissing number $k(K)$ is the maximum number of translates of $K$ such that no two translates overlap each other and each translate touches but does not overlap with $K$. In the case of $\frac{1}{2} C_{n}^{*}$, we have

$$
k\left(\frac{1}{2} C_{n}^{*}\right)=\max \left\{\begin{array}{l|l}
|D| & \begin{array}{l}
D \cup\{\mathbf{0}\} \text { is a packing set for } \frac{1}{2} C_{n}^{*} \text { and } \\
\left(\mathbf{x}+\frac{1}{2} C_{n}^{*}\right) \cap \frac{1}{2} C_{n}^{*} \neq \varnothing \text { for any } \mathbf{x} \in D
\end{array}
\end{array}\right\},
$$

and since $k(K)$ is invariant under the scaling of $K$,

$$
k\left(C_{n}^{*}\right)+1=k\left(\frac{1}{2} C_{n}^{*}\right)+1 \leqslant \gamma\left(C_{n}^{*}, \frac{1}{2}\right) .
$$

For the cross-polytope, it is known that $k\left(C_{3}^{*}\right)=18[13,18]$. This result implies that $\gamma\left(C_{3}^{*}, \frac{1}{2}\right) \geqslant 19$, however, due to the requirement that one point is the origin, it does not a priori provide an upper bound for any packing set for $\frac{1}{2} C_{3}^{*}$. An upper bound for the kissing number of any convex body $K$ is obtained from a result of Hadwiger [11],

$$
k(K) \leqslant 3^{n}-1
$$

where this inequality is an equality iff $K$ is a parallelepiped. The cross-polytope is not a parallelepiped, so $k\left(C_{3}^{*}\right) \leqslant 25$, which results in an upper bound of $\gamma\left(C_{3}^{*}, \frac{1}{2}\right) \leqslant 26$. In the other direction, a well-known result by Swinnerton-Dyer [17] says that

$$
k(K) \geqslant n^{2}+n
$$

and in the case when $K$ is a cross-polytope the lower bound has been considerably improved to

$$
k\left(C_{n}^{*}\right) \geqslant\left(\frac{9}{8}\right)^{(1-o(1)) n}
$$

by $[13]$ and to

$$
k\left(C_{n}^{*}\right) \geqslant 1.13488^{(1-o(1)) n}
$$

by [19], which is the best known asymptotic lower bound for the cross-polytope. 
We will work with values of $r$ only in the interval $\left(\frac{1}{2}, 1\right]$ unless otherwise stated. For dimension $n$ and $r \in\left(1-\frac{1}{n}, 1\right]$, the upper bound for the number of points in the crosspolytope such that the distance between any two distinct points is at least $2 r$ is linear in the dimension of the cross-polytope.

Theorem 1. Let $n \geqslant 2$, then $\gamma\left(C_{n}^{*}, r\right)=2 n$ for any $r \in\left(1-\frac{1}{n}, 1\right]$. Additionally, $\left(1-\frac{1}{n}, 1\right]$ is the largest possible interval such that $\gamma\left(C_{n}^{*}, r\right)=2 n$ for all $r$ in the interval.

In particular, $\gamma\left(C_{3}^{*}, r\right)=6$ for $r \in\left(\frac{2}{3}, 1\right]$. The next theorem is specific to the threedimensional case.

Theorem 2. In dimension 3,

(a) $\gamma\left(C_{3}^{*}, r\right)=10$ for any $r \in\left(\frac{3}{5}, \frac{2}{3}\right]$,

(b) $\gamma\left(C_{3}^{*}, r\right)=12$ for any $r \in\left(\frac{4}{7}, \frac{3}{5}\right]$, and

(c) $\gamma\left(C_{3}^{*}, r\right) \leqslant 14$ for $r \in\left(\frac{1}{2}, \frac{4}{7}\right]$.

For the case $n=3$ and $r \in\left(\frac{1}{2}, \frac{4}{7}\right]$, we could not find exact values of $\gamma\left(C_{3}^{*}, r\right)$, but we do have lower bounds. Since $\gamma\left(C_{3}^{*}, r^{\prime}\right) \geqslant \gamma\left(C_{3}^{*}, r\right)$ for $r^{\prime}<r$, it follows immediately from Theorem 2 (b) is $\gamma\left(C_{3}^{*}, r\right) \geqslant 12$ for $r \in\left(\frac{1}{2}, \frac{4}{7}\right]$. It is possible to improve this lower bound for a smaller interval of $r$.

Proposition 3. In dimension $3, \gamma\left(C_{3}^{*}, r\right) \geqslant 13$ for $r \in\left(\frac{1}{2}, \frac{6}{11}\right]$.

These lower bounds are obtained by explicit constructions. It follows from Theorems 1 and 2, Proposition 3, and the above discussion that

$$
\begin{aligned}
\gamma\left(C_{n}^{*}, r\right)=1 & \text { for } r \in(1, \infty), \\
\gamma\left(C_{n}^{*}, r\right)=2 n & \text { for } r \in\left(1-\frac{1}{n}, 1\right], \\
\gamma\left(C_{3}^{*}, r\right)=10 & \text { for } r \in\left(\frac{3}{5}, \frac{2}{3}\right], \\
\gamma\left(C_{3}^{*}, r\right)=12 & \text { for } r \in\left(\frac{4}{7}, \frac{3}{5}\right], \\
12 \leqslant \gamma\left(C_{3}^{*}, r\right) \leqslant 14 & \text { for } r \in\left(\frac{6}{11}, \frac{4}{7}\right], \\
12 \leqslant \gamma\left(C_{3}^{*}, r\right) \leqslant 14 & \text { for } r \in\left(\frac{1}{2}, \frac{6}{11}\right], \text { and } \\
19 \leqslant \gamma\left(C_{3}^{*}, r\right) \leqslant 26 & \text { for } r=\frac{1}{2} .
\end{aligned}
$$

Below is a chart of the results for dimension 3 in addition to the upper and lower bounds for $r=\frac{1}{2}$ mentioned above. 


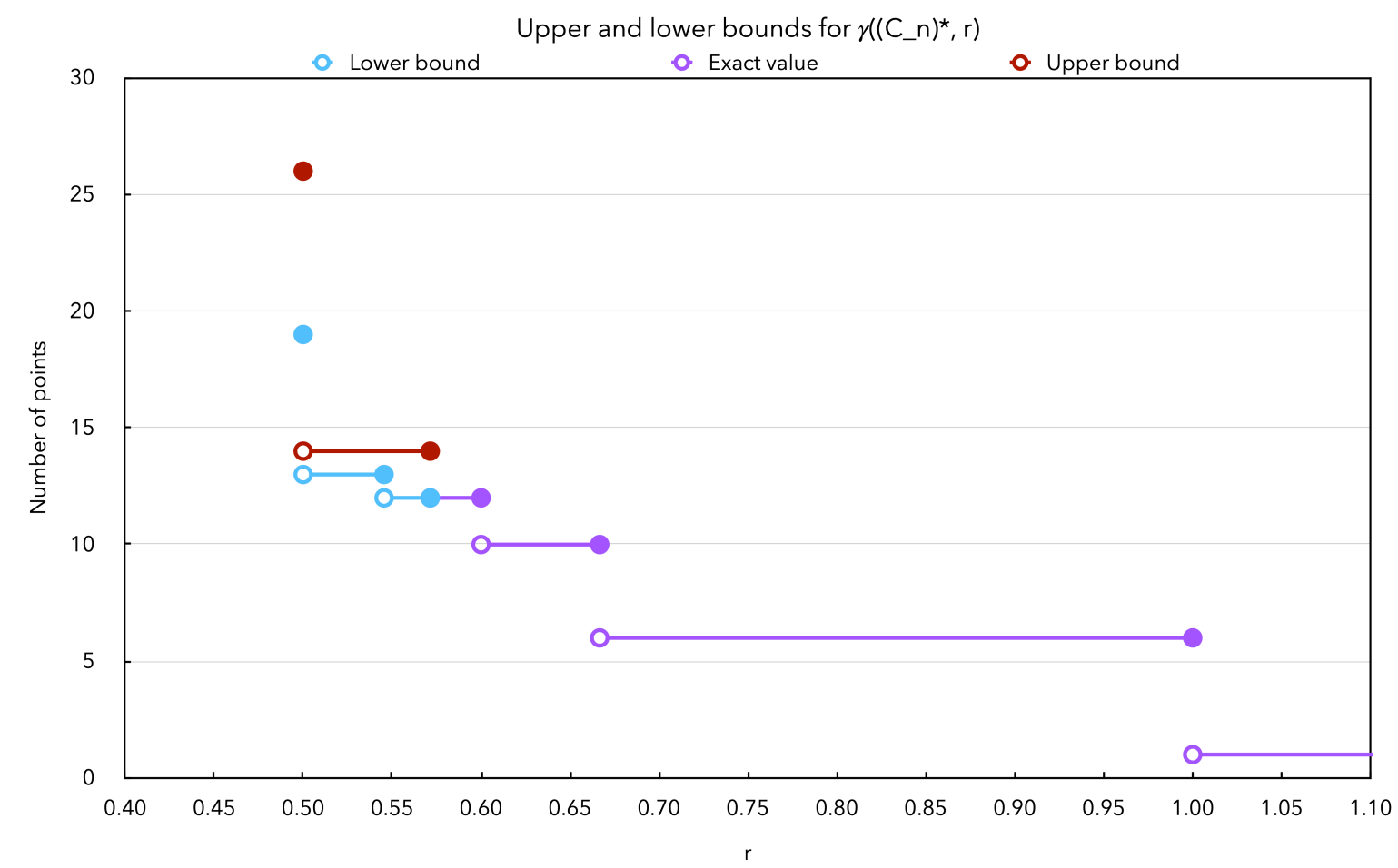

For the $n$-dimensional ball $B_{n}$, Hajós and Davenport showed that $\gamma\left(B_{n}, r\right) \leqslant n+1$ for $r>\frac{1}{\sqrt{2}}$, as noted by [6].

A closely related topic is the problem of packing a set with copies of another set. This problem has been explored mainly in dimension 2. Let $K$ and $L$ be origin-symmetric convex sets with nonempty interior, then let

$M(L, K, m):=\sup \left\{r|| D \mid=m, D \subset L\right.$, and $\|\mathbf{x}-\mathbf{y}\|_{K} \geqslant 2 r$ for any $\left.\mathbf{x}, \mathbf{y} \in D, \mathbf{x} \neq \mathbf{y}\right\}$.

From this definition, $M(L, K, m)$ and $\gamma(L, K, r)$ are related by the equation

$$
\gamma(L, K, M(L, K, m))=m \text {. }
$$

The compendium of Goodman, O'Rourke, and Tóth [8] lists known quantities of $M\left(L, B_{2}, m\right)$ for when $L$ is a square, a circle, and an equilateral triangle and various values of $m$, usually small. In three dimensions, $M\left(L, B_{3}, m\right)$ is known for small $m$ and when $L$ is a cube, a cross-polytope, and a tetrahedron [8]. A related problem of packings of squares and rectangles in squares is described in [4]. Let $B_{n}$ be the unit Euclidean ball in $n$ dimensions. Böröczky Jr. and Wintsche have obtained $M\left(C_{n}^{*}, B_{n}, m\right)$ for $n \geqslant 3$ and $m=\{3, \ldots, 2 n+1\}[1]$.

Let $K$ and $B$ be convex bodies, $s>0$, and let $D(s, K, B)$ be a packing set of $C_{n}^{*}$ such that

$|\{\mathbf{x} \in D(s, K, B) \mid K+\mathbf{x} \subseteq s B\}|$ is maximal among all packing sets of $C_{n}^{*}$. The density of the densest packing of $K$, or the packing density of $K$, is defined to be

$$
\delta(K):=\lim _{s \rightarrow \infty} \frac{|\{\mathbf{x} \in D(s, K, B) \mid K+\mathbf{x} \subseteq s B\}| \operatorname{Vol}(K)}{\operatorname{Vol}(s B)},
$$


see Definition 4 in Section 20 of [10] (page 225), and it is independent of $B$. Then we can set $B=C_{n}^{*}$ and also suppose that $K=C_{n}^{*}$. For $s>1$, since $C_{n}^{*}+\mathbf{x} \subseteq s C_{n}^{*}$ iff $\mathbf{x} \in(s-1) C_{n}^{*}$, we have

$$
\left|\left\{\mathbf{x} \in D(s, K, B) \mid C_{n}^{*}+\mathbf{x} \subseteq s C_{n}^{*}\right\}\right|=\left|\left\{\mathbf{x} \in D(s, K, B) \mid \mathbf{x} \in(s-1) C_{n}^{*}\right\}\right| .
$$

Next, scale this set by a factor of $\frac{1}{s-1}$ to get

$$
\begin{aligned}
\left|\left\{\mathbf{x} \in D(s, K, B) \mid \mathbf{x} \in(s-1) C_{n}^{*}\right\}\right| & =\left|\left\{\mathbf{x} \in \frac{1}{s-1} D(s, K, B) \mid \mathbf{x} \in C_{n}^{*}\right\}\right| \\
& =\gamma\left(C_{n}^{*}, \frac{1}{s-1}\right) .
\end{aligned}
$$

Now let $r=\frac{1}{s-1}$. It follows from the definition of the packing density that

$$
\delta\left(C_{n}^{*}\right)=\lim _{s \rightarrow \infty} \frac{\gamma\left(C_{n}^{*}, \frac{1}{s-1}\right) \operatorname{Vol}\left(C_{n}^{*}\right)}{\operatorname{Vol}\left(s C_{n}^{*}\right)}=\lim _{r \rightarrow 0} \gamma\left(C_{n}^{*}, r\right)\left(1-\frac{1}{1+r}\right)^{n} .
$$

Hence the packing density of $C_{n}^{*}$ is related to $\gamma\left(C_{n}^{*}, r\right)$ in the sense that $\gamma\left(C_{n}^{*}, r\right)\left(1-\frac{1}{1+r}\right)^{n} \sim \delta\left(C_{n}^{*}\right)$ as $r \rightarrow 0$.

We now mention some related results involving circle packings in a circle and sphere packings in a cylinder. For the problem of sphere packing inside a cylinder of fixed width in three dimensions, Fu et al. [7] predict that as the radius of the spheres approach zero, densest packings resemble the face-centered cubic lattice - a densest sphere packing in three dimensions [2] - except for the spheres that are near the walls of the cylinder. In the case of dimension two the densest circle packing is generated by the hexagonal lattice [2]. Hopkins, Stillinger, and Torquato [12] provide examples of this phenomenon for dense packings of circles inside a large circle under the condition that the large circle has the same center as one of the small circles. Schürmann $[15,16]$ has shown that under certain conditions the best finite packings of strictly convex bodies can only be obtained using nonlattice packings. Other dense arrangements of $k$ circles within a large circle include modified wedge hexagonal packings and curved hexagonal packings [12], which are the best known packings for some values of $k$ [14].

The density of an infinite packing of a convex set is the fraction of space taken up by congruent copies of the set. Fejes Tóth, Fodor, and Vígh [5] describe some upper bounds for the packing density of $C_{n}^{*}$, including an asymptotic $\delta\left(C_{n}^{*}\right) \leqslant C \cdot 0.86850^{n}$ for $n \geqslant 7$ and a fixed constant $C$. Furthermore, they conjecture that $\delta\left(C_{n}^{*}\right) \leqslant C \cdot 0.82886^{n}$.

Basic facts about convexity and the cross-polytope can be found in books such as the ones from Gruber [9], Ziegler [20], and Coxeter [3], and about packings are in Conway and Sloane [2], Gruber [9], and Zong [21]. Additional details on the kissing number are also in Zong [21].

Section 2 of this paper provides the notation and preliminaries that will be used for the rest of the text. Section 3 contains the proof of the $n$-dimensional case, Theorem 1. Section 4 proves the equalities and upper bound present in the three parts of the 
3-dimensional case, Theorem 2, introducing additional notation as needed. Theorem 3 is proved in Section 5, and finally Section 6 presents a gallery of diagrams related to these lower bounds.

\section{General notation and preliminaries}

Here we introduce notation that will be used over the course of this paper. For a given $r>0$, let $P_{n}(r) \subset C_{n}^{*}$ be a packing set of $r C_{n}^{*}$. For any polytope $K$, let vert $(K)$ be the set of its vertices. For a fixed $n \in \mathbb{N}$, define sets $V_{n}$ and $S_{n}(r)$ as follows:

$$
V_{n}:=\operatorname{vert}\left(C_{n}^{*}\right)=\left\{ \pm \mathbf{e}_{i} \mid i \in\{1, \ldots, n\}\right\}
$$

and

$$
S_{n}(r):=\left(V_{n}+2 r \operatorname{int}\left(C_{n}^{*}\right)\right) \cap C_{n}^{*} .
$$

Therefore $S_{n}(r)$ is the set of all points in $C_{n}^{*}$ that are of distance $<2 r$ from some vertex of $C_{n}^{*}$.

For $\mathbf{p} \in \mathbb{R}^{n}$ and $r>0$, we use the notation

$$
C(\mathbf{p}, r):=\left\{\mathbf{x} \in \mathbb{R}^{n} \mid\|\mathbf{x}-\mathbf{p}\|_{1}<r\right\}
$$

to denote the interior of the cross-polytope centered at $\mathbf{p}$ and scaled by the factor $r$.

The following lemma is necessary for the general $n$-dimensional case.

Lemma 4. Let $r \in(0,1]$. For each $j \in\{1, \ldots, n\}$,

$$
C_{n}^{*} \cap C\left(\mathbf{e}_{j}, 2 r\right) \subseteq \overline{C\left((1-r) \mathbf{e}_{j}, r\right)}
$$

where $\bar{X}$ is the closure of $X$, and similarly for $-\mathbf{e}_{j}$ instead of $\mathbf{e}_{j}$.

Proof. Without loss of generality we take the $\mathbf{e}_{j}$ case. Let $\mathbf{y}=\sum_{i=1}^{n} y_{i} \mathbf{e}_{i} \in C_{n}^{*} \cap C\left(\mathbf{e}_{j}, 2 r\right)$, then $\|\mathbf{y}\|_{1} \leqslant 1$ and $\left\|\mathbf{y}-\mathbf{e}_{j}\right\|_{1} \leqslant 2 r$. Then the distance from $\mathbf{y}$ to $(1-r) \mathbf{e}_{j}$ is

$$
\left\|\mathbf{y}-(1-r) \mathbf{e}_{j}\right\|_{1}=\sum_{i \neq j}\left|y_{i}\right|+\left|y_{j}+r-1\right| .
$$

If $y_{j}+r-1 \geqslant 0$ then since $\sum_{i=1}^{n}\left|y_{i}\right| \leqslant 1$,

$$
\begin{aligned}
\sum_{i \neq j}\left|y_{i}\right|+\left|y_{j}+r-1\right| & \leqslant\left(1-y_{j}\right)+y_{j}+r-1 \\
& =r .
\end{aligned}
$$

Similarly, if $y_{j}+r-1<0$ then since $\left\|\mathbf{y}-\mathbf{e}_{j}\right\|_{1} \leqslant 2 r$,

$$
\sum_{i \neq j}\left|y_{i}\right|+\left|y_{j}+r-1\right|=\sum_{i \neq j}\left|y_{i}\right|-y_{j}-r+1
$$




$$
\begin{aligned}
& =\sum_{i \neq j}\left|y_{i}\right|+\left|y_{j}-1\right|-r \\
& \leqslant 2 r-r \\
& =r .
\end{aligned}
$$

So $\left\|\mathbf{y}-(1-r) \mathbf{e}_{j}\right\|_{1} \leqslant r$, or in other words, $\mathbf{y} \in\left\{\mathbf{x} \in \mathbb{R}^{n} \mid\left\|\mathbf{x}-(1-r) \mathbf{e}_{j}\right\|_{1} \leqslant r\right\}=$ $\overline{C\left((1-r) \mathbf{e}_{j}, r\right)}$.

\section{Proof of Theorem 1: the $n$-dimensional case}

In this section we assume that $n \geqslant 2$. We will show that for any $r \in\left(1-\frac{1}{n}, 1\right]$ and any packing set $P_{n}(r)$, the number of points in $P_{n}(r) \cap S_{n}(r)$ is bounded above by the number of vertices of $C_{n}^{*}$. Then $\left|P_{n}(r)\right| \leqslant 2 n$ and this inequality is true for all $P_{n}(r)$, so $\gamma\left(C_{n}^{*}, r\right) \leqslant 2 n$. As mentioned in the introduction, the set of vertices $V_{n} \subset C_{n}^{*}$ is a packing set of $r C_{n}^{*}$, which means that $2 n$ is also a lower bound, and so $\gamma\left(C_{n}^{*}, r\right)=2 n$.

Lemma 5. Let $r \in\left(1-\frac{1}{n}, 1\right]$, then $C_{n}^{*}=S_{n}(r)$.

Proof. By definition, $S_{n}(r) \subseteq C_{n}^{*}$, and so it remains to show the reverse inclusion. Let $\mathbf{x} \in C_{n}^{*}$ and without loss of generality it can be assumed that $\mathbf{x}$ is in the convex hull of $\mathbf{0}, \mathbf{e}_{1}, \ldots, \mathbf{e}_{n}$. Then $\mathbf{x}=\sum_{i=1}^{n} x_{i} \mathbf{e}_{i}$ with $0 \leqslant x_{i} \leqslant 1$ and $\sum_{i=1}^{n} x_{i} \leqslant 1$. Then there exists some $j \in\{1, \ldots, n\}$ such that $x_{j} \geqslant \frac{1}{n} \sum_{i=1}^{n} x_{i}$, so

$$
\begin{aligned}
\left\|\mathbf{x}-\mathbf{e}_{j}\right\|_{1} & =\sum_{i=1}^{n} x_{i}-2 x_{j}+1 \\
& \leqslant\left(1-\frac{2}{n}\right) \sum_{i=1}^{n} x_{i}+1 \\
& <2 r .
\end{aligned}
$$

So every point in $C_{n}^{*}$ is within distance $2 r$ from some vertex of $C_{n}^{*}$.

The next lemma will be crucial for showing that the number of points in $P_{n}(r) \cap S_{n}(r)$ is bounded above by the number of vertices of $C_{n}^{*}$. It is a uniqueness condition which shows that if a point $\mathbf{p} \in P_{n}(r) \cap S_{n}(r)$ is close to a vertex $\mathbf{v}$ of $C_{n}^{*}$, specifically $\|\mathbf{p}-\mathbf{v}\|_{1}<2 r$, then no other point in $P_{n}(r)$ can be close to $\mathbf{v}$.

Lemma 6. Let $r \in(0,1]$. If a vertex $\mathbf{v}$ of $C_{n}^{*}$ has the property that $\mathbf{v} \in C(\mathbf{p}, 2 r) \cap C(\mathbf{q}, 2 r)$ for some $\mathbf{p}, \mathbf{q} \in P_{n}(r) \cap S_{n}(r)$, then $\mathbf{p}=\mathbf{q}$.

Proof. Without loss of generality, let $\mathbf{v}=\mathbf{e}_{j}$ for some $j \in\{1, \ldots, n\}$, then by hypothesis $\mathbf{e}_{j} \in C(\mathbf{p}, 2 r) \cap C(\mathbf{q}, 2 r)$. It suffices to show that $\|\mathbf{p}-\mathbf{q}\|_{1}<2 r$ since the distance between two distinct points in $P_{n, r}$ must be $2 r$ or greater.. Then in turn, $\mathbf{p}, \mathbf{q} \in C\left(\mathbf{e}_{j}, 2 r\right)$. Since 
$C\left(\mathbf{e}_{j}, 2 r\right)$ is open there exists a $r^{\prime}<r\left(r^{\prime}\right.$ depends on $\mathbf{p}$ and $\left.\mathbf{q}\right)$ such that $\mathbf{p}, \mathbf{q} \in C\left(\mathbf{e}_{j}, 2 r^{\prime}\right)$. Then it follows from Lemma 4 applied to $C_{n}^{*} \cap C\left(\mathbf{e}_{j}, 2 r^{\prime}\right)$ that

$$
C_{n}^{*} \cap C\left(\mathbf{e}_{j}, 2 r^{\prime}\right) \subseteq \overline{C\left(\left(1-r^{\prime}\right) \mathbf{e}_{j}, r^{\prime}\right)} \subseteq C\left(\left(1-r^{\prime}\right) \mathbf{e}_{j}, r\right),
$$

so $\|\mathbf{p}-\mathbf{q}\|_{1}<2 r$.

The following lemma will be used both here and in the 3-dimensional cases in the next section.

Lemma 7. Let $r \in(0,1]$, then

$$
\left|P_{n}(r) \cap S_{n}(r)\right| \leqslant 2 n \text {. }
$$

Proof. Define $V_{n}(r)$ by

$$
V_{n}(r)=\left\{\mathbf{v} \in V_{n} \mid \text { there exists a } \mathbf{p} \in P_{n}(r) \cap S_{n}(r) \text { such that }\|\mathbf{v}-\mathbf{p}\|_{1}<2 r\right\}
$$

(this set may be empty) and a map $f: V_{n}(r) \rightarrow P_{n}(r) \cap S_{n}(r)$ where $f(\mathbf{v})$ is the point $\mathbf{p} \in P_{n}(r) \cap S_{n}(r)$ such that $\|\mathbf{v}-\mathbf{p}\|_{1}<2 r$.

First we need to show that $f$ is well-defined. Let $\mathbf{p}, \mathbf{q} \in P_{n}(r) \cap S_{n}(r)$ be points such that $\mathbf{v} \in C(\mathbf{p}, 2 r) \cap C(\mathbf{q}, 2 r)$ for some $\mathbf{v} \in V_{n}$, then $\mathbf{p}=\mathbf{q}$ by Lemma 6 , which justifies the use of the words "the point" in the definition of $f$. From the definition of $S_{n}(r)$, every point $\mathbf{p} \in P_{n}(r) \cap S_{n}(r)$ has the property that there is some $\mathbf{v} \in V_{n}$ such that $\|\mathbf{p}-\mathbf{v}\|_{1}<2 r$, so $f$ is surjective. Both the domain and range of $f$ are finite sets, so the cardinality of the range can be bounded above by

$$
\left|P_{n}(r) \cap S_{n}(r)\right|=|\operatorname{Ran}(f)| \leqslant|\operatorname{Dom}(f)| \leqslant\left|V_{n}(r)\right|=2 n,
$$

completing the proof.

Now we prove Theorem 1. With the preparation above, the proof is mostly a matter of putting together earlier lemmas.

Proof of Theorem 1. Assume that $P_{n}(r)$ is nonempty, otherwise $\left|P_{n}(r)\right|=0$ and there is nothing to prove. Since $r \in\left(1-\frac{1}{n}, 1\right]$, it follows from Lemma 5 that $C_{n}^{*}=S_{n}$, so $\left|P_{n}(r) \cap S_{n}(r)\right|$ is nonempty. Then Lemma 7 shows that $\left|P_{n}(r)\right|=\left|P_{n}(r) \cap S_{n}(r)\right| \leqslant 2 n$. This inequality holds for any $P_{n}(r)$, so

$$
\gamma\left(C_{n}^{*}, r\right) \leqslant 2 n \quad \text { for } n \geqslant 2 \text { and } r \in\left(1-\frac{1}{n}, 1\right] .
$$

The upper bound of $2 n$ is achieved by $V_{n}=\left\{ \pm \mathbf{e}_{i} \mid i \in\{1, \ldots, n\}\right\}$ as a packing set of $r C_{n}^{*}$, so

$$
\gamma\left(C_{n}^{*}, r\right)=2 n \quad \text { for } n \geqslant 2 \text { and } r \in\left(1-\frac{1}{n}, 1\right] .
$$

The interval $r \in\left(1-\frac{1}{n}, 1\right]$ cannot be extended in either direction, because $\gamma\left(C_{n}^{*}, r\right)=1$ for $r>1$ and in Proposition 15 we construct a packing set of $r C_{n}^{*}$, for $r \leqslant 1-\frac{1}{n}$, with $2 n+2$ points in $C_{n}^{*}$. For such $r, S_{n}(r) \subsetneq C_{n}^{*}$ and specifically the centroid of each facet is not in $S_{n}(r)$ (cf. Subsection 4.1), so the set consisting of the $2 n$ vertices of $C_{n}^{*}$ and the two centroids on opposing facets of $C_{n}^{*}$ is a packing set of $r C_{n}^{*}$. Therefore, $r \in\left(1-\frac{1}{n}, 1\right]$ is the largest possible interval such that $\gamma\left(C_{n}^{*}, r\right)=2 n$ is true. 


\section{Proof of Theorem 2 (the 3-dimensional case)}

When $r \leqslant \frac{2}{3}$ the set $S_{3}(r)$ no longer covers all of $C_{3}^{*}$, so unlike the $n$-dimensional case above, the proofs for the three-dimensional cases require consideration of the remainder $C_{3}^{*} \backslash S_{3}(r)$.

\subsection{Notation and preliminaries for dimension 3}

Here we collect some lemmas and notation for the three-dimensional cases. Let $r \in\left[\frac{1}{2}, \frac{2}{3}\right]$. Recall that

$$
V_{3}=\operatorname{vert}\left(C_{3}^{*}\right)=\left\{ \pm \mathbf{e}_{1}, \pm \mathbf{e}_{2}, \pm \mathbf{e}_{3}\right\}
$$

and

$$
\begin{aligned}
S_{3}(r) & =\left(V_{3}+2 r \operatorname{int}\left(C_{n}^{*}\right)\right) \cap C_{n}^{*} \\
& =\bigcup_{i=1}^{3}\left(C\left(\mathbf{e}_{i}, 2 r\right) \cup C\left(-\mathbf{e}_{i}, 2 r\right)\right) .
\end{aligned}
$$

For any $\sigma_{1}, \sigma_{2}, \sigma_{3} \in\{-1,1\}$, define the following subsets of $\mathbb{R}^{3}$ :

$V\left(r,\left(\sigma_{1}, \sigma_{2}, \sigma_{3}\right)\right):=\left\{\left(\begin{array}{c}\sigma_{1}(2 r-1) \\ \sigma_{2}(2 r-1) \\ \sigma_{3}(2 r-1)\end{array}\right),\left(\begin{array}{c}\sigma_{1}(1-r) \\ \sigma_{2}(1-r) \\ \sigma_{3}(2 r-1)\end{array}\right),\left(\begin{array}{c}\sigma_{1}(1-r) \\ \sigma_{2}(2 r-1) \\ \sigma_{3}(1-r)\end{array}\right),\left(\begin{array}{c}\sigma_{1}(2 r-1) \\ \sigma_{2}(1-r) \\ \sigma_{3}(1-r)\end{array}\right)\right\}$.

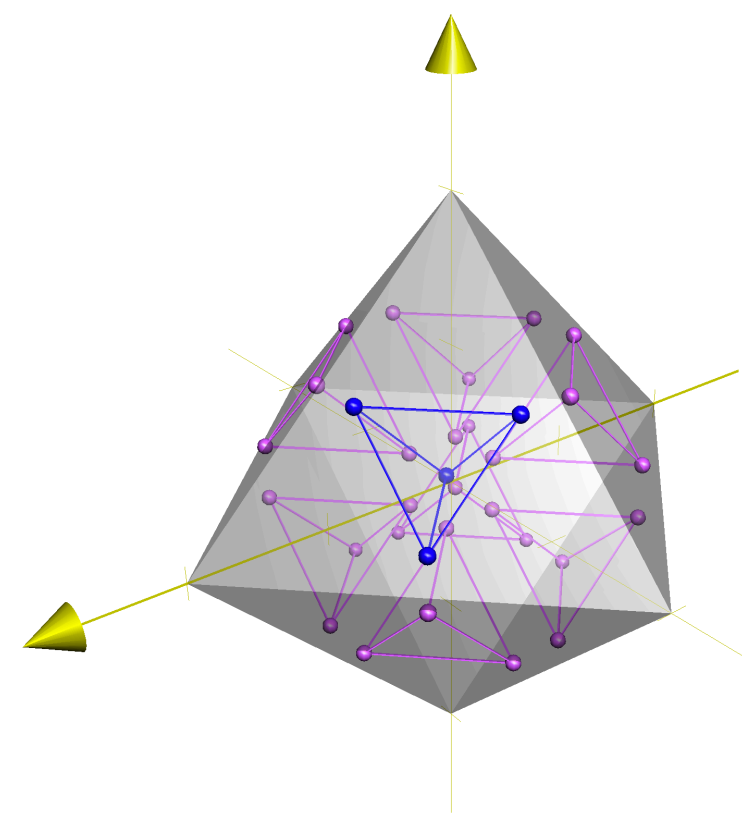

Figure 1: The grey cross-polytope is the set $C_{3}^{*}$, the blue spheres are the points of $V\left(\frac{11}{20},(1,1,1)\right)$, and the purple spheres are the points of $V\left(\frac{11}{20},\left(\sigma_{1}, \sigma_{2}, \sigma_{3}\right)\right)$ for $\sigma_{1}, \sigma_{2}, \sigma_{3} \in$ $\{-1,1\}$ and not all equal to 1 . 
The endpoints of the range $r \in\left[\frac{1}{2}, \frac{2}{3}\right]$ are $\frac{2}{3}$ and $\frac{1}{2}$. For $r=\frac{2}{3}$ the set reduces to

$$
V\left(\frac{2}{3},(1,1,1)\right)=\left\{\left(\begin{array}{c}
\frac{1}{3} \\
\frac{1}{3} \\
\frac{1}{3}
\end{array}\right)\right\}
$$

the centroid of the facet conv $\left\{\mathbf{e}_{1}, \mathbf{e}_{2}, \mathbf{e}_{3}\right\}$, and when $r=\frac{1}{2}$ the set is

$$
V\left(\frac{1}{2},(1,1,1)\right)=\left\{\left(\begin{array}{l}
0 \\
0 \\
0
\end{array}\right),\left(\begin{array}{c}
\frac{1}{2} \\
\frac{1}{2} \\
0
\end{array}\right),\left(\begin{array}{c}
\frac{1}{2} \\
0 \\
\frac{1}{2}
\end{array}\right),\left(\begin{array}{c}
0 \\
\frac{1}{2} \\
\frac{1}{2}
\end{array}\right)\right\},
$$

which contains the midpoints of the edges of the facet conv $\left\{\mathbf{e}_{1}, \mathbf{e}_{2}, \mathbf{e}_{3}\right\}$.

Subsets defined using midpoints of edges are used to solve the related problems of finding upper bounds for $k\left(C_{3}^{*}\right)$ and $M\left(C_{n}^{*}, B_{n}, m\right)$ for some values of $n$ and $m$. To find the kissing number of the cross-polytope, Larman and Zong [13] divided the boundary of the cross-polytope into the union of 18 subsets including sets of the form

$$
\operatorname{relint}\left(\left(\frac{1}{2} \mathbf{m}+\frac{1}{2} C_{3}^{*}\right) \cap C_{3}^{*}\right)
$$

where $\mathbf{m}$ is a midpoint of an edge in $V_{3}$, and showed that each subset could contain the center of at most one cross-polytope, resulting in $k\left(C_{3}^{*}\right) \leqslant 18$. Another method to prove that $k\left(C_{3}^{*}\right) \leqslant 18$ was used by Talata [18], who showed that any packing set achieving a kissing number of 18 must consist of six points on the vertices, six points on the midpoints of the edges of two opposing facets, and the remaining points on the hexagon passing through the midpoints of the other edges. Böröczky and Wintsche [1] use sets defined by vertices and midpoints of edges to determine an upper bound for $M\left(C_{n}^{*}, B_{n}, m\right)$ where $n \geqslant 3$ and $m \in\{4, \ldots, 2 n\}$.

For a packing set $P_{3}(r)$ and a set $\operatorname{conv}\left(V\left(r,\left(\sigma_{1}, \sigma_{2}, \sigma_{3}\right)\right)\right), \sigma_{1}, \sigma_{2}, \sigma_{3} \in\{-1,1\}$, call $\operatorname{conv}(V)$ a blocked set of $P_{3}(r)$ if $\operatorname{conv}\left(V\left(r,\left(\sigma_{1}, \sigma_{2}, \sigma_{3}\right)\right)\right)$ does not contain any points of $P_{3}(r)$.

First we show that $C_{3}^{*} \backslash S_{3}(r)$ can be written in terms of $V\left(r,\left(\sigma_{1}, \sigma_{2}, \sigma_{3}\right)\right)$.

Lemma 8. Let $r \in\left(\frac{1}{2}, \frac{2}{3}\right]$. For any $\sigma_{1}, \sigma_{2}, \sigma_{3} \in\{-1,1\}$, define the following subsets of $C_{3}^{*}$ :

$$
R\left(r,\left(\sigma_{1}, \sigma_{2}, \sigma_{3}\right)\right):=\left(C_{3}^{*} \backslash S_{3}(r)\right) \cap\left\{\mathbf{x} \in \mathbb{R}^{3} \mid \sigma_{1} x_{1}, \sigma_{2} x_{2}, \sigma_{3} x_{3} \geqslant 0\right\} .
$$

Then $R\left(r,\left(\sigma_{1}, \sigma_{2}, \sigma_{3}\right)\right)=\operatorname{conv}\left(V\left(r,\left(\sigma_{1}, \sigma_{2}, \sigma_{3}\right)\right)\right)$ and

$$
C_{3}^{*} \backslash S_{3}(r)=\bigcup_{\sigma_{1}, \sigma_{2}, \sigma_{3} \in\{-1,1\}} \operatorname{conv}\left(V\left(r,\left(\sigma_{1}, \sigma_{2}, \sigma_{3}\right)\right)\right) .
$$

Proof. Without loss of generality, assume that $\sigma_{1}=\sigma_{2}=\sigma_{3}=1$, and we will show that $R(r,(1,1,1))=\operatorname{conv}(V(r,(1,1,1)))$. The set $R(r,(1,1,1))$ is the subset of the unit cross-polytope with all nonnegative coordinates and excluding the sets $C\left(\mathbf{e}_{i}, 2 r\right)$ 
for $i \in\{1,2,3\}$, and the set $\operatorname{conv}(V(r,(1,1,1)))$ is the intersection of the inequalities $-x_{1}-x_{2}+x_{3} \geqslant-(2 r-1), x_{1}-x_{2}-x_{3} \leqslant-(2 r-1),-x_{1}+x_{2}-x_{3} \leqslant-(2 r-1)$, and $x_{1}+x_{2}+x_{3} \leqslant 1$, since the four points in $V(r,(1,1,1))$ satisfy each inequality. We will show that $R(r,(1,1,1))$ is also the intersection of these inequalities.

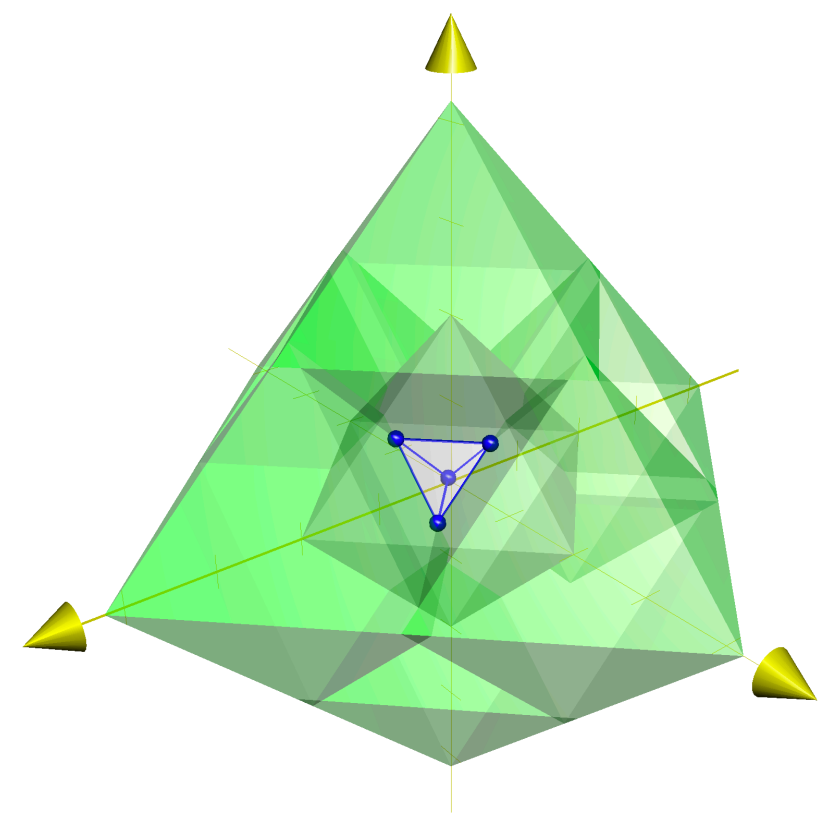

Figure 2: The grey cross-polytope is the set $C_{3}^{*}$, the green cross-polytopes are the sets $\overline{C\left(\mathbf{x}, \frac{11}{20}\right)}$ for $\mathbf{x} \in V_{3}$, and the blue spheres are the points of $V\left(\frac{11}{20},(1,1,1)\right)$.

Let $\mathbf{x} \in R(r,(1,1,1))$. Then $\mathbf{x} \in C_{3}^{*}$ so $x_{1}+x_{2}+x_{3} \leqslant 1$, and in addition, $\mathbf{x} \notin C\left(\mathbf{e}_{1}, 2 r\right)$ so $\left|x_{1}-1\right|+\left|x_{2}\right|+\left|x_{3}\right| \geqslant 2 r$ implies that $x_{1}-x_{2}-x_{3} \leqslant-(2 r-1)$. Similarly, $\mathbf{x} \notin C\left(\mathbf{e}_{2}, 2 r\right)$ and $\mathbf{x} \notin C\left(\mathbf{e}_{3}, 2 r\right)$ so $-x_{1}+x_{2}-x_{3} \leqslant-(2 r-1)$ and $x_{1}+x_{2}-x_{3} \geqslant 2 r-1$. That proves $R(r,(1,1,1)) \subseteq \operatorname{conv}(V(r,(1,1,1)))$.

For the converse, let $\mathbf{x} \in \operatorname{conv}(V(r,(1,1,1)))$. Since $\frac{1}{2} \leqslant r \leqslant \frac{2}{3}$, both $2 r-1 \geqslant 0$ and $1-r \geqslant 0$, so the four points in $V(r,(1,1,1))$ all have nonnegative coordinates. Also, $\|\mathbf{v}\|_{1} \leqslant 1$ for all $\mathbf{v} \in V(r,(1,1,1))$, and since $\mathbf{x}$ is in the convex hull of $V(r,(1,1,1))$, it is also true that $x_{1}+x_{2}+x_{3} \leqslant 1$. Then $x_{1}, x_{2}, x_{3} \geqslant 0$ and $x_{1}+x_{2}+x_{3} \leqslant 1$ imply that $\mathbf{x} \in C_{n}^{*}$. Also, $\mathbf{x}$ satisfies $x_{1}-x_{2}-x_{3} \leqslant-(2 r-1)$, then $\left|x_{1}-1\right|+\left|x_{2}\right|+\left|x_{3}\right| \geqslant 2 r$, which holds because $x_{2}, x_{3} \geqslant 0$ and $0 \leqslant x_{1} \leqslant \frac{1}{2}$, so $\mathbf{x} \notin C\left(\mathbf{e}_{1}, 2 r\right)$. Similarly, $-x_{1}+x_{2}-x_{3} \leqslant-(2 r-1)$ and $-x_{1}+x_{2}-x_{3} \leqslant-(2 r-1)$ so $\mathbf{x} \notin C\left(\mathbf{e}_{2}, 2 r\right)$ and $\mathbf{x} \notin C\left(\mathbf{e}_{3}, 2 r\right)$. Hence

$$
\mathbf{x} \in\left(C_{3}^{*} \cap\left\{x_{1}, x_{2}, x_{3} \geqslant 0\right\}\right) \backslash\left(\bigcup_{i=1}^{3} C\left(\mathbf{e}_{i}, 2 r\right)\right)=R(r,(1,1,1)) .
$$

To complete the proof of the lemma, note that for any $\mathbf{x} \in \mathbb{R}^{3}$, let $\sigma_{i}=\frac{x_{i}}{\left|x_{i}\right|}$ if $x_{i} \neq 0$ and $\sigma_{i}=1$ if $x_{i}=0$, then $\sigma_{1} x_{1}, \sigma_{2} x_{2}, \sigma_{3} x_{3} \geqslant 0$, so $C_{3}^{*} \backslash S_{3}(r)$ is indeed covered by all the 
$R\left(r,\left(\sigma_{1}, \sigma_{2}, \sigma_{3}\right)\right), \sigma_{1}, \sigma_{2}, \sigma_{3} \in\{-1,1\}$, resulting in

$$
C_{3}^{*} \backslash S_{3}(r)=\bigcup_{\sigma_{1}, \sigma_{2}, \sigma_{3} \in\{-1,1\}} R\left(r,\left(\sigma_{1}, \sigma_{2}, \sigma_{3}\right)\right)=\bigcup_{\sigma_{1}, \sigma_{2}, \sigma_{3} \in\{-1,1\}} \operatorname{conv}\left(V\left(r,\left(\sigma_{1}, \sigma_{2}, \sigma_{3}\right)\right)\right) .
$$

From this lemma, the cross-polytope $C_{3}^{*}$ is the union of $S_{3}(r)$ and the eight regions $\operatorname{conv}\left(V\left(r,\left(\sigma_{1}, \sigma_{2}, \sigma_{3}\right)\right)\right)$. By Lemma 7 ,

$$
\left|P_{3}(r) \cap S_{3}(r)\right| \leqslant 6,
$$

but for $r \in\left(0, \frac{2}{3}\right]$, some points of $P_{3}(r)$ may be contained in one or more of the sets $\operatorname{conv}\left(V\left(r,\left(\sigma_{1}, \sigma_{2}, \sigma_{3}\right)\right)\right)$.

We will use an approach that has similarities to Larman and Zong [13] and Böröczky and Wintsche [1] in that the maximum distance between any two points in $\operatorname{conv}\left(V\left(r,\left(\sigma_{1}, \sigma_{2}, \sigma_{3}\right)\right)\right)$ is less than $2 r$. Then each conv $\left(V\left(r,\left(\sigma_{1}, \sigma_{2}, \sigma_{3}\right)\right)\right)$ can contain at most one point of $P_{3}(r)$, and the number of points of $P_{3}(r)$ in $S_{3}^{*} \backslash S_{3}(r)$ is bounded above by 8 (see Subsection 4.4).

Lemma 9. Let $r \in\left(\frac{1}{2}, \frac{2}{3}\right]$ and $\sigma_{1}, \sigma_{2}, \sigma_{3} \in\{-1,1\}$. For any two points $\mathbf{x}, \mathbf{y} \in \operatorname{conv}\left(V\left(r,\left(\sigma_{1}, \sigma_{2}, \sigma_{3}\right)\right)\right),\|\mathbf{x}-\mathbf{y}\|_{1}<2 r$.

Proof. Without loss of generality, let $\sigma_{1}=\sigma_{2}=\sigma_{3}=1$, then $\mathbf{x}, \mathbf{y} \in \operatorname{conv}(V(r,(1,1,1)))$. It suffices to show that the distance between any two points in $V(r,(1,1,1))$ is less than $2 r$, then the conclusion for all points in $\operatorname{conv}(V(r,(1,1,1)))$ follows by the convexity of $\operatorname{conv}(V(r,(1,1,1)))$. We also assume that the two points are distinct. Suppose that neither point is $(2 r-1,2 r-1,2 r-1)^{\top}$, where $\mathbf{v}^{\top}$ is the transpose of $\mathbf{v}$, then both points are permutations of $(1-r, 1-r, 2 r-1)^{\top}$, so the distance between the two points is

$$
\|\mathbf{x}-\mathbf{y}\|_{1}=4-6 r<2 r .
$$

If one of the points is $(2 r-1,2 r-1,2 r-1)^{\top}$, then the other point must be a permutation of $(1-r, 1-r, 2 r-1)^{\top}$, so the distance between the two points is also

$$
\|\mathbf{x}-\mathbf{y}\|_{1}=4-6 r<2 r .
$$

When $r \in\left(\frac{4}{7}, \frac{2}{3}\right]$, the required minimum distance between points of $P_{3}(r)$ is large enough so that the presence of a point of $P_{3}(r)$ in one set $\operatorname{conv}\left(V\left(r\left(\sigma_{1}, \sigma_{2}, \sigma_{3}\right)\right)\right)$ may imply that another set $\operatorname{conv}\left(V\left(r\left(\sigma_{1}^{\prime}, \sigma_{2}^{\prime}, \sigma_{3}^{\prime}\right)\right)\right),\left(\sigma_{1}, \sigma_{2}, \sigma_{3}\right) \neq\left(\sigma_{1}^{\prime}, \sigma_{2}^{\prime}, \sigma_{3}^{\prime}\right)$, cannot contain any points in $P_{3}(r)$. Then it is possible to obtain an upper bound of 12 , and the proof in Subsection 4.3 uses a more complicated argument involving the position of $\mathbf{p}$ in $V\left(r,\left(\sigma_{1}, \sigma_{2}, \sigma_{3}\right)\right)$. In Subsection 4.2 we prove that when $r \in\left(\frac{3}{5}, \frac{2}{3}\right]$, a point $\mathbf{p} \in$ $P_{3}(r) \cap V\left(r,\left(\sigma_{1}, \sigma_{2}, \sigma_{3}\right)\right)$ implies that three other sets of the form conv $\left(V\left(r\left(\sigma_{1}, \sigma_{2}, \sigma_{3}\right)\right)\right)$ cannot contain any points in $P_{3}(r)$. 


\subsection{Proof of Theorem 2 (a): the $r \in\left(\frac{3}{5}, \frac{2}{3}\right]$ case}

Lemma 10. Let $r \in\left(\frac{3}{5}, \frac{2}{3}\right]$ and $\mathbf{p} \in P_{3}(r)$. If $\mathbf{p} \in \operatorname{conv}\left(V\left(r,\left(\sigma_{1}, \sigma_{2}, \sigma_{3}\right)\right)\right)$ for any $\sigma_{1}, \sigma_{2}, \sigma_{3} \in\{1,1\}$, then $\operatorname{conv}\left(V\left(r,\left(-\sigma_{1}, \sigma_{2}, \sigma_{3}\right)\right)\right)$, $\operatorname{conv}\left(V\left(r,\left(\sigma_{1},-\sigma_{2}, \sigma_{3}\right)\right)\right)$, and $\operatorname{conv}\left(V\left(r,\left(\sigma_{1}, \sigma_{2},-\sigma_{3}\right)\right)\right)$ are blocked sets of $P_{3}(r)$.

Proof. Without loss of generality, assume that $\sigma_{1}=\sigma_{2}=\sigma_{3}=1$. To show that $\operatorname{conv}\left(V\left(r,\left(-\sigma_{1}, \sigma_{2}, \sigma_{3}\right)\right)\right)$ is a blocked set of $P_{3}(r)$, it suffices to show that $\left\|\mathbf{p}-\mathbf{y}^{\prime}\right\|_{1}<2 r$ for all

$$
\mathbf{y}^{\prime} \in V(r,(-1,1,1))=\left\{\left(\begin{array}{c}
-(2 r-1) \\
2 r-1 \\
2 r-1
\end{array}\right),\left(\begin{array}{c}
-(1-r) \\
1-r \\
2 r-1
\end{array}\right),\left(\begin{array}{c}
-(1-r) \\
2 r-1 \\
1-r
\end{array}\right),\left(\begin{array}{c}
-(2 r-1) \\
1-r \\
1-r
\end{array}\right)\right\}
$$

then by the convexity of $V(r,(-1,1,1))$, the statement $\|\mathbf{p}-\mathbf{y}\|_{1}<2 r$ holds true for any $\mathbf{y} \in \operatorname{conv}(V(r,(-1,1,1)))$. Since $-(1-r) \leqslant p_{1} \leqslant-(2 r-1)$ and $2 r-1 \leqslant p_{2}, p_{3} \leqslant 1-r$, we have

$$
\begin{aligned}
&\left\|\mathbf{p}-\left(\begin{array}{c}
-(2 r-1) \\
2 r-1 \\
2 r-1
\end{array}\right)\right\|_{1}=-p_{1}+p_{2}+p_{3}-6 r+3 \leqslant 6-9 r<2 r, \\
&\left\|\mathbf{p}-\left(\begin{array}{c}
-(1-r) \\
1-r \\
2 r-1
\end{array}\right)\right\|_{1}=p_{1}-p_{2}+p_{3}-4 r+3<2 r, \\
&\left\|\mathbf{p}-\left(\begin{array}{c}
-(1-r) \\
2 r-1 \\
1-r
\end{array}\right)\right\|_{1}=p_{1}+p_{2}-p_{3}-4 r+3<2 r,
\end{aligned}
$$

and

$$
\left\|\mathbf{p}-\left(\begin{array}{c}
-(2 r-1) \\
1-r \\
1-r
\end{array}\right)\right\|_{1}=-p_{1}-p_{2}-p_{3}-4 r+3<2 r
$$

By the symmetry of $V(r,(-1,1,1)), V(r,(1,-1,1))$, and $V(r,(1,1,-1))$, it follows that $\|\mathbf{p}-\mathbf{y}\|_{1}<2 r$ for any $\mathbf{y} \in V(r,(-1,1,1)) \cup V(r,(1,-1,1)) \cup V(r,(1,1,-1))$, and so these three sets are blocked sets of $P_{3}(r)$.

If $P_{3}(r) \cap\left(C_{3}^{*} \backslash S_{3}(r)\right)=\varnothing$ then trivially every set of the form conv $\left(V\left(r,\left(\sigma_{1}, \sigma_{2}, \sigma_{3}\right)\right)\right)$, $\sigma_{1}, \sigma_{2}, \sigma_{3} \in\{-1,1\}$, is a blocked set of $P_{3}(r)$. Otherwise, the above lemma implies that for any given $P_{3}(r)$, three of the eight sets of the form $\operatorname{conv}\left(V\left(r,\left(\sigma_{1}, \sigma_{2}, \sigma_{3}\right)\right)\right)$ are blocked sets of $P_{3}(r)$. Therefore,

$$
\left|P_{3}(r) \cap\left(C_{3}^{*} \backslash S_{3}(r)\right)\right| \leqslant 5 .
$$

However, it is possible to lower the 5 to a 4 with the following argument.

Lemma 11. Let $r \in\left(\frac{3}{5}, \frac{2}{3}\right]$. Then for any $P_{3}(r)$, there exist at least four blocked sets of $P_{3}(r)$. 
Proof. Let $\mathbf{p} \in P_{3}(r) \cap\left(C_{3}^{*} \backslash S_{3}(r)\right)$. Without loss of generality assume that there is a $\mathbf{p} \in$ $V(r,(1,1,1))$. Then by Lemma $10, V(r,(-1,1,1)), V(r,(1,-1,1))$, and $V(r,(1,1,-1))$ are blocked sets of $P_{3}(r)$. Consider the set $V(r,(-1,-1,-1))$. If it is a blocked set, then there is nothing more to prove. If it is not, then again by Lemma $10, V(r,(1,-1,-1))$, $V(r,(-1,1,-1))$, and $V(r,(-1,-1,1))$ are blocked sets of $P_{3}(r)$, resulting in a total of six blocked sets.

Lemma 12. Let $r \in\left(\frac{1}{2}, \frac{2}{3}\right]$ and suppose that $\left|P_{3}(r) \cap\left(C_{3}^{*} \backslash S_{3}(r)\right)\right| \leqslant b$ for all $P_{3}(r)$, then

$$
\gamma\left(C_{3}^{*}, r\right) \leqslant 6+b
$$

Proof. Split up $P_{3}(r)$ into $P_{3}(r) \cap S_{3}(r)$ and $P_{3}(r) \cap\left(C_{3}^{*} \backslash S_{3}(r)\right)$, then by Lemma 7 , $\left|P_{3}(r) \cap S_{3}(r)\right| \leqslant 6$. So

$$
\begin{aligned}
\left|P_{3}(r)\right| & \leqslant\left|P_{3}(r) \cap S_{3}(r)\right|+\left|P_{3}(r) \cap\left(C_{3}^{*} \backslash S_{3}(r)\right)\right| \\
& \leqslant 6+b .
\end{aligned}
$$

By hypothesis, this inequality holds for any $P_{3}(r)$, so

$$
\gamma\left(C_{3}^{*}, r\right) \leqslant 6+b .
$$

With the lemma above we prove Theorem 2 (a), (b), and (c).

Proof of Theorem 2 (a). Let $r \in\left(\frac{3}{5}, \frac{2}{3}\right]$. By Lemma 11 there are at least four blocked sets of $P_{3}(r)$, and from the definition of a blocked set at least four of the eight sets $\operatorname{conv}\left(V\left(r,\left(\sigma_{1}, \sigma_{2}, \sigma_{3}\right)\right)\right), \sigma_{1}, \sigma_{2}, \sigma_{3} \in\{-1,1\}$, cannot contain points of $P_{3}(r)$. Then $\left|P_{3}(r) \cap\left(C_{3}^{*} \backslash S_{3}(r)\right)\right| \leqslant 4$, so by Lemma 12 ,

$$
\gamma\left(C_{3}^{*}, r\right) \leqslant 10 .
$$

From Proposition 16 below, there is a 10-point packing set for $r C_{3}^{*}$ contained in $C_{3}^{*}$, so in fact

$$
\gamma\left(C_{3}^{*}, r\right)=10
$$

\subsection{Proof of Theorem 2 (b): the $r \in\left(\frac{4}{7}, \frac{3}{5}\right]$ case}

The following additional notation will be used in this section. For each $r>0$ and $\sigma_{1}, \sigma_{2}, \sigma_{3} \in\{-1,1\}$, define the following sets $V\left(r,\left(\sigma_{1}, \sigma_{2}, \sigma_{3}\right)\right)$ :

$$
\begin{gathered}
V\left(r,\left(\sigma_{1}, \sigma_{2}, \sigma_{3}\right), 1\right) \\
:=\left\{\left(\begin{array}{l}
\sigma_{1}(2 r-1) \\
\sigma_{2}(2 r-1) \\
\sigma_{3}(2 r-1)
\end{array}\right),\left(\begin{array}{c}
\sigma_{1}(2 r-1) \\
\sigma_{2}(1-r) \\
\sigma_{3}(1-r)
\end{array}\right),\left(\begin{array}{c}
\sigma_{1} \frac{1}{2} r \\
\sigma_{2}(1-r) \\
\sigma_{3} \frac{1}{2} r
\end{array}\right),\left(\begin{array}{c}
\sigma_{1} \frac{1}{2} r \\
\sigma_{2} \frac{1}{2} r \\
\sigma_{3}(1-r)
\end{array}\right), \frac{1}{3}\left(\begin{array}{l}
\sigma_{1} \\
\sigma_{2} \\
\sigma_{3}
\end{array}\right)\right\}, \\
V\left(r,\left(\sigma_{1}, \sigma_{2}, \sigma_{3}\right), 2\right) \\
:=\left\{\left(\begin{array}{l}
\sigma_{1}(2 r-1) \\
\sigma_{2}(2 r-1) \\
\sigma_{3}(2 r-1)
\end{array}\right),\left(\begin{array}{c}
\sigma_{1}(1-r) \\
\sigma_{2}(2 r-1) \\
\sigma_{3}(1-r)
\end{array}\right),\left(\begin{array}{c}
\sigma_{1}(1-r) \\
\sigma_{2} \frac{1}{2} r \\
\sigma_{3} \frac{1}{2} r
\end{array}\right),\left(\begin{array}{c}
\sigma_{1} \frac{1}{2} r \\
\sigma_{2} \frac{1}{2} r \\
\sigma_{3}(1-r)
\end{array}\right), \frac{1}{3}\left(\begin{array}{l}
\sigma_{1} \\
\sigma_{2} \\
\sigma_{3}
\end{array}\right)\right\},
\end{gathered}
$$




$$
\begin{aligned}
V & \left(r,\left(\sigma_{1}, \sigma_{2}, \sigma_{3}\right), 3\right) \\
& :=\left\{\left(\begin{array}{l}
\sigma_{1}(2 r-1) \\
\sigma_{2}(2 r-1) \\
\sigma_{3}(2 r-1)
\end{array}\right),\left(\begin{array}{c}
\sigma_{1}(1-r) \\
\sigma_{2}(1-r) \\
\sigma_{3}(2 r-1)
\end{array}\right),\left(\begin{array}{c}
\sigma_{1}(1-r) \\
\sigma_{2} \frac{1}{2} r \\
\sigma_{3} \frac{1}{2} r
\end{array}\right),\left(\begin{array}{c}
\sigma_{1} \frac{1}{2} r \\
\sigma_{2}(1-r) \\
\sigma_{3} \frac{1}{2} r
\end{array}\right), \frac{1}{3}\left(\begin{array}{l}
\sigma_{1} \\
\sigma_{2} \\
\sigma_{3}
\end{array}\right)\right\} .
\end{aligned}
$$

They have the property that

$$
\bigcup_{i=1}^{3} \operatorname{conv}\left(V\left(r,\left(\sigma_{1}, \sigma_{2}, \sigma_{3}\right), i\right)\right)=\operatorname{conv}\left(V\left(r,\left(\sigma_{1}, \sigma_{2}, \sigma_{3}\right)\right)\right)
$$

and the numbering of these subsets is so that the set $V\left(r,\left(\sigma_{1}, \sigma_{2}, \sigma_{3}\right), i\right)$ contains the point in the set

$$
\left\{\left(\begin{array}{c}
\sigma_{1}(1-r) \\
\sigma_{2}(1-r) \\
\sigma_{3}(2 r-1)
\end{array}\right),\left(\begin{array}{c}
\sigma_{1}(1-r) \\
\sigma_{2}(2 r-1) \\
\sigma_{3}(1-r)
\end{array}\right),\left(\begin{array}{c}
\sigma_{1}(2 r-1) \\
\sigma_{2}(1-r) \\
\sigma_{3}(1-r)
\end{array}\right)\right\} \subsetneq V\left(r,\left(\sigma_{1}, \sigma_{2}, \sigma_{3}\right)\right)
$$

that is furthest away from the vertex $\sigma_{i} \mathbf{e}_{i}$.

Lemma 13. Let $r \in\left(\frac{4}{7}, \frac{3}{5}\right]$ and $\mathbf{p} \in P_{3}(r)$. If $\mathbf{x} \in \operatorname{conv}\left(V\left(r,\left(\sigma_{1}, \sigma_{2}, \sigma_{3}\right)\right)\right)$ then there is a blocked set conv $\left(V\left(r,\left(\sigma_{1}^{\prime}, \sigma_{2}^{\prime}, \sigma_{3}^{\prime}\right)\right)\right)$ of $P_{3}(r)$, with $\left(\sigma_{1}, \sigma_{2}, \sigma_{3}\right)$ and $\left(\sigma_{1}^{\prime}, \sigma_{2}^{\prime}, \sigma_{3}^{\prime}\right)$ differing by exactly one coordinate.

Proof. Without loss of generality, assume that $\sigma_{1}=\sigma_{2}=\sigma_{3}=1$, then $\mathbf{p}$ is in one of the subsets $\operatorname{conv}(V(r,(1,1,1), i))$ for $i \in\{1,2,3\}$. Assume that $\mathbf{p} \in \operatorname{conv}(V(r,(1,1,1), 1))$ and write $\mathbf{p}=\sum_{i=1}^{3} p_{i} \mathbf{e}_{i}$. We will show that $\|\mathbf{x}-\mathbf{y}\|_{1}<2 r$ for any $\mathbf{x} \in V(r,(1,1,1), 1)$ and $\mathbf{y} \in V(r,(-1,1,1))$, and then by the convexity of $\operatorname{conv}(V(r,(1,1,1), 1))$ and $\operatorname{conv}(V(r,(-1,1,1)))$, it follows that $\|\mathbf{p}-\mathbf{y}\|_{1}<2 r$ for any $\mathbf{y} \in \operatorname{conv}(V(r,(-1,1,1)))$, which shows that conv $(V(r,(-1,1,1)))$ is a blocked set of $P_{3}(r)$. This approach is similar to the proof of the previous lemma, but the same approach cannot be used here as that proof requires $r>\frac{3}{5}$.

There are 20 different combinations of points but not all of them need to be explicitly checked. To keep track of the cases, we use the following grid: 


\begin{tabular}{|c|c|c|c|c|c|}
\hline & \multicolumn{4}{|c|}{ Elements of $V(r,(-1,1,1))$} \\
\hline & & $\left(\begin{array}{c}-(2 r-1) \\
2 r-1 \\
2 r-1\end{array}\right)$ & $\left(\begin{array}{c}-(1-r) \\
1-r \\
2 r-1\end{array}\right)$ & $\left(\begin{array}{c}-(1-r) \\
2 r-1 \\
1-r\end{array}\right)$ & $\left(\begin{array}{c}-(2 r-1) \\
1-r \\
1-r\end{array}\right)$ \\
\hline \multirow{5}{*}{$\begin{array}{c}\text { Elements of } \\
V(r,(1,1,1), 1)\end{array}$} & $\left(\begin{array}{l}2 r-1 \\
2 r-1 \\
2 r-1\end{array}\right)$ & Case 1 & Case 2 & Case 3 & Case 4 \\
\hline & $\left(\begin{array}{c}2 r-1 \\
1-r \\
1-r\end{array}\right)$ & Case 5 & Case 6 & Case 7 & Case 8 \\
\hline & $\left(\begin{array}{c}\frac{1}{2} r \\
1-r\end{array}\right)$ & Case 9 & Case 10 & Case 11 & Case 12 \\
\hline & $\left(\begin{array}{c}\frac{1}{2} r \\
\frac{1}{2} r \\
1-r\end{array}\right)$ & Case 13 & Case 14 & Case 15 & Case 16 \\
\hline & $\left(\begin{array}{l}\frac{1}{3} \\
\frac{1}{3}\end{array}\right)$ & Case 17 & Case 18 & Case 19 & Case 20 \\
\hline
\end{tabular}

For each $k \in\{1, \ldots, 20\}$, case $k$ corresponds to the calculation of $\|\mathbf{x}-\mathbf{y}\|_{1}$, where $\mathbf{x}$ is the element of $V(r,(1,1,1), 1)$ in the same row as $k$ and $\mathbf{y}$ is the element of $V(r,(-1,1,1))$ in the same column as $k$. For example,

$$
\left\|\left(\begin{array}{c}
2 r-1 \\
2 r-1 \\
2 r-1
\end{array}\right)-\left(\begin{array}{c}
-(2 r-1) \\
2 r-1 \\
2 r-1
\end{array}\right)\right\|_{1}
$$

corresponds to case 1 below.

1. Cases 1 and 8:

$$
\begin{aligned}
& \left\|\left(\begin{array}{c}
2 r-1 \\
2 r-1 \\
2 r-1
\end{array}\right)-\left(\begin{array}{c}
-(2 r-1) \\
2 r-1 \\
2 r-1
\end{array}\right)\right\|_{1}=4 r-2<2 r, \\
& \left\|\left(\begin{array}{c}
2 r-1 \\
1-r \\
1-r
\end{array}\right)-\left(\begin{array}{c}
-(2 r-1) \\
1-r \\
1-r
\end{array}\right)\right\|_{1}=4 r-2<2 r .
\end{aligned}
$$

2. Cases 2, 3, 4, 5, 6, 7, 9, 10, 13, 15, and 17:

$$
\left\|\left(\begin{array}{c}
2 r-1 \\
2 r-1 \\
2 r-1
\end{array}\right)-\left(\begin{array}{c}
-(1-r) \\
1-r \\
2 r-1
\end{array}\right)\right\|_{1}=2-2 r<2 r
$$




$$
\begin{aligned}
& \left\|\left(\begin{array}{l}
2 r-1 \\
2 r-1 \\
2 r-1
\end{array}\right)-\left(\begin{array}{c}
-(1-r) \\
2 r-1 \\
1-r
\end{array}\right)\right\|_{1}=2-2 r<2 r, \\
& \left\|\left(\begin{array}{c}
2 r-1 \\
2 r-1 \\
2 r-1
\end{array}\right)-\left(\begin{array}{c}
-(2 r-1) \\
1-r \\
1-r
\end{array}\right)\right\|_{1}=2-2 r<2 r \\
& \left\|\left(\begin{array}{c}
2 r-1 \\
1-r \\
1-r
\end{array}\right)-\left(\begin{array}{c}
-(2 r-1) \\
2 r-1 \\
2 r-1
\end{array}\right)\right\|_{1}=2-2 r<2 r \\
& \left\|\left(\begin{array}{c}
2 r-1 \\
1-r \\
1-r
\end{array}\right)-\left(\begin{array}{c}
-(1-r) \\
1-r \\
2 r-1
\end{array}\right)\right\|_{1}=2-2 r<2 r, \\
& \left\|\left(\begin{array}{c}
2 r-1 \\
1-r \\
1-r
\end{array}\right)-\left(\begin{array}{c}
-(1-r) \\
2 r-1 \\
1-r
\end{array}\right)\right\|_{1}=2-2 r<2 r, \\
& \left\|\left(\begin{array}{c}
\frac{1}{2} r \\
1-r \\
\frac{1}{2} r
\end{array}\right)-\left(\begin{array}{c}
-(2 r-1) \\
2 r-1 \\
2 r-1
\end{array}\right)\right\|_{1}=2-2 r<2 r, \\
& \left\|\left(\begin{array}{c}
\frac{1}{2} r \\
1-r \\
\frac{1}{2} r
\end{array}\right)-\left(\begin{array}{c}
-(1-r) \\
1-r \\
2 r-1
\end{array}\right)\right\|_{1}=2-2 r<2 r, \\
& \left\|\left(\begin{array}{c}
\frac{1}{2} r \\
\frac{1}{2} r \\
1-r
\end{array}\right)-\left(\begin{array}{c}
-(2 r-1) \\
2 r-1 \\
2 r-1
\end{array}\right)\right\|_{1}=2-2 r<2 r \\
& \left\|\left(\begin{array}{c}
\frac{1}{2} r \\
\frac{1}{2} r \\
1-r
\end{array}\right)-\left(\begin{array}{c}
-(1-r) \\
2 r-1 \\
1-r
\end{array}\right)\right\|_{1}=2-2 r<2 r \\
& \left\|\left(\begin{array}{c}
\frac{1}{3} \\
\frac{1}{3} \\
\frac{1}{3}
\end{array}\right)-\left(\begin{array}{c}
-(2 r-1) \\
2 r-1 \\
2 r-1
\end{array}\right)\right\|_{1}=2-2 r<2 r .
\end{aligned}
$$

3. Cases 11 and 14:

$$
\begin{aligned}
& \left\|\left(\begin{array}{c}
\frac{1}{2} r \\
1-r \\
\frac{1}{2} r
\end{array}\right)-\left(\begin{array}{c}
-(1-r) \\
2 r-1 \\
1-r
\end{array}\right)\right\|_{1}=4-5 r<2 r, \\
& \left\|\left(\begin{array}{c}
\frac{1}{2} r \\
\frac{1}{2} r \\
1-r
\end{array}\right)-\left(\begin{array}{c}
-(1-r) \\
1-r \\
2 r-1
\end{array}\right)\right\|_{1}=4-5 r<2 r .
\end{aligned}
$$


4. Cases 12 and 16:

$$
\begin{aligned}
& \left\|\left(\begin{array}{c}
\frac{1}{2} r \\
1-r \\
\frac{1}{2} r
\end{array}\right)-\left(\begin{array}{c}
-(2 r-1) \\
1-r \\
1-r
\end{array}\right)\right\|_{1}=r<2 r \\
& \left\|\left(\begin{array}{c}
\frac{1}{2} r \\
\frac{1}{2} r \\
1-r
\end{array}\right)-\left(\begin{array}{c}
-(2 r-1) \\
1-r \\
1-r
\end{array}\right)\right\|_{1}=r<2 r .
\end{aligned}
$$

5. Cases 18 and 19:

$$
\begin{aligned}
& \left\|\left(\begin{array}{c}
\frac{1}{3} \\
\frac{1}{3} \\
\frac{1}{3}
\end{array}\right)-\left(\begin{array}{c}
-(1-r) \\
1-r \\
2 r-1
\end{array}\right)\right\|_{1}=\frac{10}{3}-4 r<2 r, \\
& \left\|\left(\begin{array}{c}
\frac{1}{3} \\
\frac{1}{3} \\
\frac{1}{3}
\end{array}\right)-\left(\begin{array}{c}
-(1-r) \\
2 r-1 \\
1-r
\end{array}\right)\right\|_{1}=\frac{10}{3}-4 r<2 r .
\end{aligned}
$$

6. Case 20:

$$
\left\|\left(\begin{array}{c}
\frac{1}{3} \\
\frac{1}{3} \\
\frac{1}{3}
\end{array}\right)-\left(\begin{array}{c}
-(2 r-1) \\
1-r \\
1-r
\end{array}\right)\right\|_{1}=\frac{2}{3}<2 r .
$$

Hence conv $(V(r,(-1,1,1)))$ is a blocked set of $P_{3}(r)$. By symmetry, if $\mathbf{p} \in \operatorname{conv}(V(r,(1,1,1), 2))$ or $\mathbf{p} \in \operatorname{conv}(V(r,(1,1,1), 3))$ then calculations similar to the above can be performed with $\mathbf{y} \in \operatorname{conv}(V(r,(1,-1,1)))$ or $\mathbf{y} \in \operatorname{conv}(V(r,(1,1,-1)))$ respectively.

Using the above lemma and the same argument as after Lemma 10 in the last subsection, we have

$$
\left|P_{3}(r) \cap\left(C_{3}^{*} \backslash S_{3}(r)\right)\right| \leqslant 7 .
$$

However, as in the previous subsection it is possible to lower the 7 to a 6 with the following argument.

Lemma 14. Let $r \in\left(\frac{4}{7}, \frac{3}{5}\right]$. Then for any $P_{3}(r)$, there exist at least two blocked sets of $P_{3}(r)$.

Proof. Let conv $\left(V\left(r,\left(\sigma_{1}, \sigma_{2}, \sigma_{3}\right)\right)\right), \sigma_{1}, \sigma_{2}, \sigma_{3} \in\{-1,1\}$, be a blocked set of $P_{3}(r)$ and consider the set $\operatorname{conv}\left(V\left(r,\left(-\sigma_{1},-\sigma_{2},-\sigma_{3}\right)\right)\right)$. If $\operatorname{conv}\left(V\left(r,\left(-\sigma_{1},-\sigma_{2},-\sigma_{3}\right)\right)\right)$ is a blocked set of $P_{3}(r)$ then we are done. Otherwise, by Lemma 13 there must be a blocked set $\operatorname{conv}\left(V\left(r,\left(\sigma_{1}^{\prime}, \sigma_{2}^{\prime}, \sigma_{3}^{\prime}\right)\right)\right)$ of $P_{3}(r)$ such that $\left(-\sigma_{1},-\sigma_{2},-\sigma_{3}\right)$ and $\left(\sigma_{1}^{\prime}, \sigma_{2}^{\prime}, \sigma_{3}^{\prime}\right)$ differ by exactly one coordinate. Then $\left(\sigma_{1}^{\prime}, \sigma_{2}^{\prime}, \sigma_{3}^{\prime}\right) \neq\left(\sigma_{1}, \sigma_{2}, \sigma_{3}\right)$, which means that $\operatorname{conv}\left(V\left(r,\left(\sigma_{1}, \sigma_{2}, \sigma_{3}\right)\right)\right)$ and conv $\left(V\left(r,\left(\sigma_{1}^{\prime}, \sigma_{2}^{\prime}, \sigma_{3}^{\prime}\right)\right)\right)$ are two distinct blocked sets of $P_{3}(r)$. 
The proof of Theorem 2 (b) is virtually identical to the proof of Theorem 2 (a).

Proof of Theorem $2(b)$. Let $r \in\left(\frac{4}{7}, \frac{3}{5}\right]$. By Lemma 14, Lemma 12, and the 12-point packing set for $r C_{3}^{*}$ from Proposition 17, we have

$$
\gamma\left(C_{3}^{*}, r\right)=12 .
$$

\subsection{Proof of Theorem 2 (c): the $r \in\left(\frac{1}{2}, \frac{4}{7}\right]$ case}

Before we prove Theorem 2 (c), we remark that an immediate consequence of Lemma 9 is that

$$
\left|P_{3}(r) \cap \operatorname{conv}\left(V\left(r,\left(\sigma_{1}, \sigma_{2}, \sigma_{3}\right)\right)\right)\right| \leqslant 1
$$

for all $\sigma_{1}, \sigma_{2}, \sigma_{3} \in\{-1,1\}$, which means that

$$
\begin{aligned}
\left|P_{3}(r) \cap\left(C_{3}^{*} \backslash S_{3}(r)\right)\right| & =\left|P_{3}(r) \cap\left(\bigcup_{\sigma_{1}, \sigma_{2}, \sigma_{3} \in\{-1,1\}} \operatorname{conv}\left(V\left(r,\left(\sigma_{1}, \sigma_{2}, \sigma_{3}\right)\right)\right)\right)\right| \\
& \leqslant \sum_{\sigma_{1}, \sigma_{2}, \sigma_{3} \in\{-1,1\}}\left|P_{3}(r) \cap \operatorname{conv}\left(V\left(r,\left(\sigma_{1}, \sigma_{2}, \sigma_{3}\right)\right)\right)\right| \\
& \leqslant 8 .
\end{aligned}
$$

Proof of Theorem 2 (c). Let $r \in\left(\frac{1}{2}, \frac{4}{7}\right]$. By the above remark and Lemma 12, we have

$$
\gamma\left(C_{3}^{*}, r\right) \leqslant 14 \text {. }
$$

We are not able to find the exact value of $\gamma\left(C_{3}^{*}, r\right)$ for such $r$, but some lower bounds are in Section 5.

\section{Constructive lower bounds including the proof of Prop. 3}

In contrast to the upper bounds, the lower bounds are all obtained by explicit constructions of points in the cross-polytope. For $n=3$ and $r \in\left(\frac{1}{2}, \frac{2}{3}\right]$, all of the constructions shown here contain the six points of $V_{3}$ and the remaining points are in the union of the eight sets conv $\left(V\left(r,\left(\sigma_{1}, \sigma_{2}, \sigma_{3}\right)\right)\right)$. There are no claims of uniqueness made here; more than one set of points may achieve the lower bounds of Theorems 2 and 3. The proofs of these propositions amount to checking that the distance between any two distinct points in the packing set is at least $2 r$, and the calculations can be performed by hand or using a computer.

Proposition 15. Let $\mathbf{q}_{n}=\left(\frac{1}{n}, \ldots, \frac{1}{n}\right)^{\top} \in \mathbb{R}^{n}$. Then $V_{n} \cup\left\{ \pm \mathbf{q}_{n}\right\} \subset C_{3}^{*}$ and for $r \in$ $\left(0,1-\frac{1}{n}\right]$,

$V_{n} \cup\left\{ \pm \mathbf{q}_{n}\right\}=\left\{\left(\begin{array}{c}1 \\ 0 \\ 0 \\ \vdots \\ 0\end{array}\right),\left(\begin{array}{c}-1 \\ 0 \\ 0 \\ \vdots \\ 0\end{array}\right),\left(\begin{array}{c}0 \\ 1 \\ 0 \\ \vdots \\ 0\end{array}\right),\left(\begin{array}{c}0 \\ -1 \\ 0 \\ \vdots \\ 0\end{array}\right), \ldots,\left(\begin{array}{c}0 \\ 0 \\ \vdots \\ 0 \\ 1\end{array}\right),\left(\begin{array}{c}0 \\ 0 \\ \vdots \\ 0 \\ -1\end{array}\right)\right\} \cup \frac{1}{n}\left\{\left(\begin{array}{c}1 \\ 1 \\ \vdots \\ 1 \\ 1\end{array}\right),-\left(\begin{array}{c}1 \\ 1 \\ \vdots \\ 1 \\ 1\end{array}\right)\right\}$ 
is a packing set of $r C_{n}^{*}$.

Proof. Any points $\mathbf{x}, \mathbf{y} \in V_{n} \cup\left\{ \pm \mathbf{q}_{n}\right\}, \mathbf{x} \neq \mathbf{y}$, have the property that $\|\mathbf{x}\|_{1} \leqslant 1$ and $\|\mathbf{x}-\mathbf{y}\|_{1} \geqslant 2\left(1-\frac{1}{n}\right)$, so $V_{n} \cup\left\{ \pm \mathbf{q}_{n}\right\} \subset C_{3}^{*}$ is a packing set of $r C_{n}^{*}$ for $r \leqslant 1-\frac{1}{n}$.

Proposition 16. Let

$$
Q_{10}=\frac{1}{3}\left\{\left(\begin{array}{l}
1 \\
1 \\
1
\end{array}\right),\left(\begin{array}{c}
-1 \\
-1 \\
1
\end{array}\right),\left(\begin{array}{c}
-1 \\
1 \\
-1
\end{array}\right),\left(\begin{array}{c}
1 \\
-1 \\
-1
\end{array}\right)\right\} .
$$

Then $V_{3} \cup Q_{10} \subset C_{3}^{*}$ and for $r \in\left(0, \frac{2}{3}\right]$,

$$
\begin{aligned}
V_{3} \cup Q_{10}= & \left\{\left(\begin{array}{l}
1 \\
0 \\
0
\end{array}\right),\left(\begin{array}{c}
-1 \\
0 \\
0
\end{array}\right),\left(\begin{array}{l}
0 \\
1 \\
0
\end{array}\right),\left(\begin{array}{c}
0 \\
-1 \\
0
\end{array}\right),\left(\begin{array}{l}
0 \\
0 \\
1
\end{array}\right),\left(\begin{array}{c}
0 \\
0 \\
-1
\end{array}\right)\right\} \\
& \cup \frac{1}{3}\left\{\left(\begin{array}{l}
1 \\
1 \\
1
\end{array}\right),\left(\begin{array}{c}
-1 \\
-1 \\
1
\end{array}\right),\left(\begin{array}{c}
-1 \\
1 \\
-1
\end{array}\right),\left(\begin{array}{c}
1 \\
-1 \\
-1
\end{array}\right)\right\}
\end{aligned}
$$

is a packing set of $r C_{3}^{*}$.

Proof. Any points $\mathbf{x}, \mathbf{y} \in V_{3} \cup Q_{10}, \mathbf{x} \neq \mathbf{y}$, have the property that $\|\mathbf{x}\|_{1} \leqslant 1$ and $\|\mathbf{x}-\mathbf{y}\|_{1} \geqslant \frac{4}{3}$, so $V_{3} \cup Q_{10} \subset C_{3}^{*}$ is a packing set of $r C_{n}^{*}$ for $r \leqslant \frac{2}{3}$.

Proposition 17. Let

$$
Q_{12}^{+}=\frac{1}{5}\left\{\left(\begin{array}{l}
2 \\
2 \\
1
\end{array}\right),\left(\begin{array}{c}
-2 \\
1 \\
2
\end{array}\right),\left(\begin{array}{c}
1 \\
-2 \\
2
\end{array}\right)\right\} .
$$

Then $V_{3} \cup Q_{12}^{+} \cup\left(-Q_{12}^{+}\right) \subset C_{3}^{*}$ and for $r \in\left(0, \frac{3}{5}\right]$,

$$
\begin{aligned}
V_{3} \cup Q_{12}^{+} \cup\left(-Q_{12}^{+}\right)= & \left\{\left(\begin{array}{l}
1 \\
0 \\
0
\end{array}\right),\left(\begin{array}{c}
-1 \\
0 \\
0
\end{array}\right),\left(\begin{array}{l}
0 \\
1 \\
0
\end{array}\right),\left(\begin{array}{c}
0 \\
-1 \\
0
\end{array}\right),\left(\begin{array}{l}
0 \\
0 \\
1
\end{array}\right),\left(\begin{array}{c}
0 \\
0 \\
-1
\end{array}\right)\right\} \\
& \cup \frac{1}{5}\left\{\left(\begin{array}{l}
2 \\
2 \\
1
\end{array}\right),\left(\begin{array}{c}
-2 \\
1 \\
2
\end{array}\right),\left(\begin{array}{c}
1 \\
-2 \\
2
\end{array}\right)\right\} \cup-\frac{1}{5}\left\{\left(\begin{array}{l}
2 \\
2 \\
1
\end{array}\right),\left(\begin{array}{c}
-2 \\
1 \\
2
\end{array}\right),\left(\begin{array}{c}
1 \\
-2 \\
2
\end{array}\right)\right\}
\end{aligned}
$$

is a packing set of $r C_{3}^{*}$.

Proof. Any points $\mathbf{x}, \mathbf{y} \in V_{3} \cup Q_{12}^{+} \cup\left(-Q_{12}^{+}\right), \mathbf{x} \neq \mathbf{y}$, have the property that $\|\mathbf{x}\|_{1} \leqslant 1$ and $\|\mathbf{x}-\mathbf{y}\|_{1} \geqslant \frac{6}{5}$, so $V_{3} \cup Q_{12}^{+} \cup\left(-Q_{12}^{+}\right) \subset C_{3}^{*}$ is a packing set of $r C_{n}^{*}$ for $r \leqslant \frac{3}{5}$.

Finally we consider the case $r \in\left(0, \frac{6}{11}\right]$. The construction below differs from the previous configurations as there appear to be no obvious large-scale symmetries. 
Proposition 18. Let

$$
Q_{13}=\frac{1}{11}\left\{\left(\begin{array}{c}
-1 \\
5 \\
5
\end{array}\right),\left(\begin{array}{c}
5 \\
-1 \\
5
\end{array}\right),\left(\begin{array}{c}
5 \\
5 \\
-1
\end{array}\right),\left(\begin{array}{c}
-5 \\
-2 \\
4
\end{array}\right),\left(\begin{array}{c}
-5 \\
4 \\
-2
\end{array}\right),\left(\begin{array}{c}
4 \\
-2 \\
-5
\end{array}\right),\left(\begin{array}{l}
-3 \\
-5 \\
-3
\end{array}\right)\right\} .
$$

Then $V_{3} \cup Q_{13} \subset C_{3}^{*}$ and for $r \leqslant \frac{6}{11}$,

$$
\begin{aligned}
V_{3} \cup Q_{13}= & \left\{\left(\begin{array}{l}
1 \\
0 \\
0
\end{array}\right),\left(\begin{array}{c}
-1 \\
0 \\
0
\end{array}\right),\left(\begin{array}{l}
0 \\
1 \\
0
\end{array}\right),\left(\begin{array}{c}
0 \\
-1 \\
0
\end{array}\right),\left(\begin{array}{l}
0 \\
0 \\
1
\end{array}\right),\left(\begin{array}{c}
0 \\
0 \\
-1
\end{array}\right)\right\} \\
& \cup \frac{1}{11}\left\{\left(\begin{array}{c}
-1 \\
5 \\
5
\end{array}\right),\left(\begin{array}{c}
5 \\
-1 \\
5
\end{array}\right),\left(\begin{array}{c}
5 \\
5 \\
-1
\end{array}\right),\left(\begin{array}{c}
-5 \\
-2 \\
4
\end{array}\right),\left(\begin{array}{c}
-5 \\
4 \\
-2
\end{array}\right),\left(\begin{array}{c}
4 \\
-2 \\
-5
\end{array}\right),\left(\begin{array}{l}
-3 \\
-5 \\
-3
\end{array}\right)\right\}
\end{aligned}
$$

is a packing set of $r C_{3}^{*}$.

Proof. Any points $\mathbf{x}, \mathbf{y} \in V_{3} \cup Q_{13}, \mathbf{x} \neq \mathbf{y}$, have the property that $\|\mathbf{x}\|_{1} \leqslant 1$ and $\|\mathbf{x}-\mathbf{y}\|_{1} \geqslant \frac{12}{11}$, so $V_{3} \cup Q_{13} \subset C_{3}^{*}$ is a packing set of $r C_{n}^{*}$ for $r \leqslant \frac{6}{11}$.

We do not know if this result can be improved, either in the sense of a 13-point configuration for some $r>\frac{6}{11}$ or a 14-point configuration for $r=\frac{6}{11}$. Regarding the first avenue for potential improvement, the upper end of the range $r \in\left(0, \frac{6}{11}\right]$ cannot be raised without moving the points of $V_{3} \cup Q_{13}$. As for the second, according to the comments before the proof of Theorem 2 (c), at most eight points in any $P_{3}\left(\frac{6}{11}\right)$ can be in the union $\bigcup_{\sigma_{1}, \sigma_{2}, \sigma_{3} \in\{-1,1\}} \operatorname{conv}\left(V\left(\frac{6}{11},\left(\sigma_{1}, \sigma_{2}, \sigma_{3}\right)\right)\right)$, with at most one point in each $\operatorname{conv}\left(V\left(\frac{6}{11},\left(\sigma_{1}, \sigma_{2}, \sigma_{3}\right)\right)\right)$. The packing set $V_{3} \cup Q_{13}$ contains points in each set of the form $\operatorname{conv}\left(V\left(\frac{6}{11},\left(\sigma_{1}, \sigma_{2}, \sigma_{3}\right)\right)\right), \sigma_{1}, \sigma_{2}, \sigma_{3} \in\{-1,1\}$, except for conv $\left(V\left(\frac{6}{11},(1,1,1)\right)\right)$, see Figure 6.6. Since

$$
V\left(\frac{6}{11},(1,1,1)\right)=\left\{\left(\begin{array}{l}
0 \\
0 \\
0
\end{array}\right), \frac{1}{11}\left(\begin{array}{l}
5 \\
5 \\
1
\end{array}\right), \frac{1}{11}\left(\begin{array}{l}
5 \\
1 \\
5
\end{array}\right), \frac{1}{11}\left(\begin{array}{l}
1 \\
5 \\
5
\end{array}\right)\right\}
$$

and the distances from each point in this set to $\frac{1}{11}(-1,5,5)^{\top} \in V_{3} \cup Q_{13}$ are

$$
\begin{gathered}
\left\|\left(\begin{array}{l}
0 \\
0 \\
0
\end{array}\right)-\frac{1}{11}\left(\begin{array}{c}
-1 \\
5 \\
5
\end{array}\right)\right\|_{1}=1<\frac{12}{11}, \\
\left\|\frac{1}{11}\left(\begin{array}{l}
5 \\
5 \\
1
\end{array}\right)-\frac{1}{11}\left(\begin{array}{c}
-1 \\
5 \\
5
\end{array}\right)\right\|_{1}=\frac{10}{11}<\frac{12}{11}, \\
\left\|\frac{1}{11}\left(\begin{array}{l}
5 \\
1 \\
5
\end{array}\right)-\frac{1}{11}\left(\begin{array}{c}
-1 \\
5 \\
5
\end{array}\right)\right\|_{1}=\frac{10}{11}<\frac{12}{11},
\end{gathered}
$$




$$
\left\|\frac{1}{11}\left(\begin{array}{l}
1 \\
5 \\
5
\end{array}\right)-\frac{1}{11}\left(\begin{array}{c}
-1 \\
5 \\
5
\end{array}\right)\right\|_{1}=\frac{2}{11}<\frac{12}{11}
$$

it follows that the distance from any point in $\operatorname{conv}\left(V\left(\frac{6}{11},(1,1,1)\right)\right)$ to $\frac{1}{11}(-1,5,5)^{\top}$ is less than $\frac{12}{11}$. Similar calculations can be performed for the distance from any point in $\operatorname{conv}\left(V\left(\frac{6}{11},(1,1,1)\right)\right)$ to $\frac{1}{11}(5,-1,5)^{\top}$ and $\frac{1}{11}(5,5,-1)^{\top}$. Therefore, without moving the points in the subset

$$
\frac{1}{11}\left\{\left(\begin{array}{c}
-1 \\
5 \\
5
\end{array}\right),\left(\begin{array}{c}
5 \\
-1 \\
5
\end{array}\right),\left(\begin{array}{c}
5 \\
5 \\
-1
\end{array}\right)\right\} \subset V_{3} \cup Q_{13}
$$

$\operatorname{conv}\left(V\left(\frac{6}{11},(1,1,1)\right)\right)$ cannot contain any points of $P_{3}\left(\frac{6}{11}\right)$ and a 14-point packing set of $\frac{6}{11} C_{3}^{*}$ is not possible.

Proof of Proposition 3. By Proposition 18, the set $V_{3} \cup Q_{13}$ is a subset of $C_{n}^{*}$ with 13 points and is a packing set for $r C_{3}^{*}$ where $r \in\left(\frac{1}{2}, \frac{6}{11}\right]$. Therefore

$$
\gamma\left(C_{3}^{*}, r\right) \geqslant 13 \quad \text { for } r \in\left(\frac{1}{2}, \frac{6}{11}\right] .
$$

\section{Diagrams of cross-polytope packings}

Below are graphs showing the unit cross-polytope with cross-polytopes of radius $r$ around each point of $V_{3}, V_{3} \cup Q_{10}, V_{3} \cup Q_{12}^{+} \cup\left(-Q_{12}^{+}\right)$, and $V_{3} \cup Q_{13}$. For each diagram except the first one, the value of $r$ in the diagram is the largest possible for that configuration of points. In each diagram the grey cross-polytope in the middle is $C_{3}^{*}$.

$\boldsymbol{V}_{\mathbf{3}}: \mathbf{6}$ points in $\boldsymbol{C}_{\mathbf{3}}^{*} \quad V_{3}$ is a packing set of $r C_{3}^{*}$ for all $0<r \leqslant 1$.

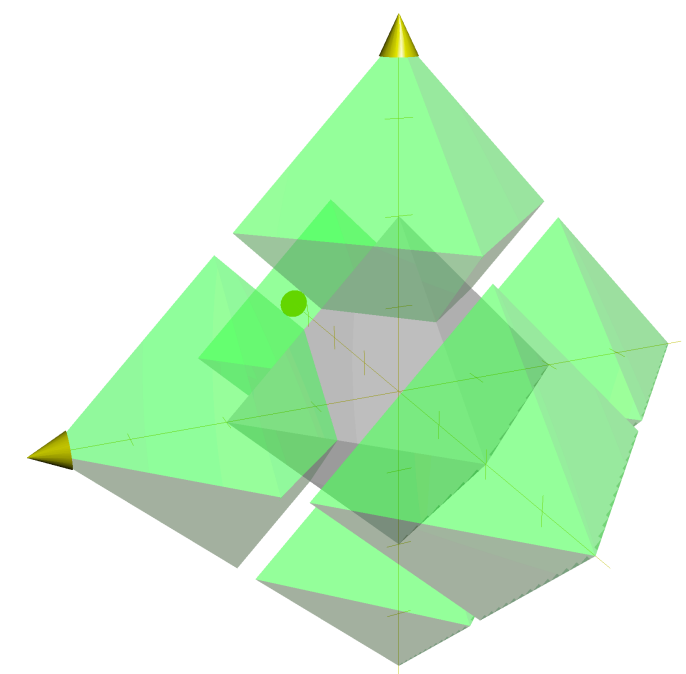

Figure 3: The green cross-polytopes represent the sets $\overline{C\left(\mathbf{x}, \frac{9}{10}\right)}$ for $\mathbf{x} \in V_{3}$. 
$\boldsymbol{V}_{\mathbf{3}} \cup \boldsymbol{Q}_{\mathbf{1 0}}: 10$ points in $\boldsymbol{C}_{\mathbf{3}}^{*} \quad V_{3} \cup Q_{10}$ is a packing set of $r C_{3}^{*}$ for all $0<r \leqslant \frac{2}{3}$.
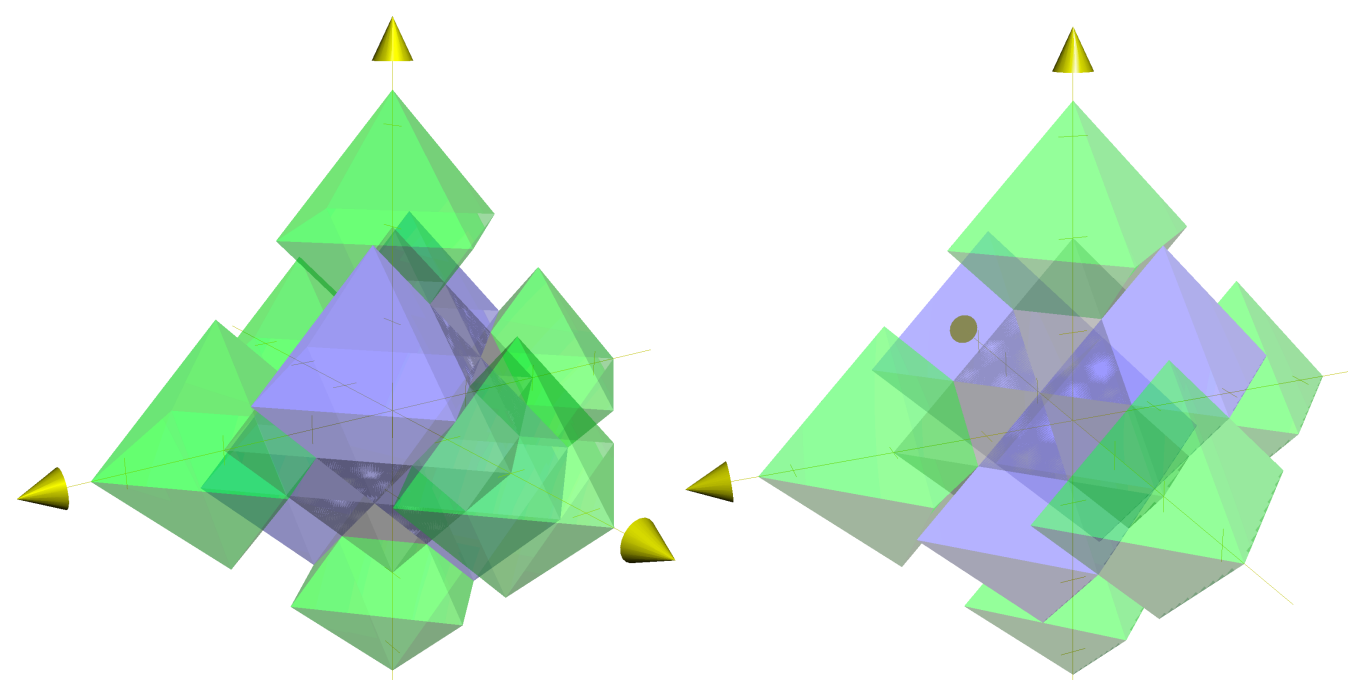

Figure 4: The green cross-polytopes represent the sets $\overline{C\left(\mathbf{x}, \frac{2}{3}\right)}$ for $\mathbf{x} \in V_{3}$ and the blue cross-polytopes represent the sets $\overline{C\left(\mathbf{y}, \frac{2}{3}\right)}$ for $\mathbf{y} \in Q_{10}$.

$\boldsymbol{V}_{\mathbf{3}} \cup \boldsymbol{Q}_{\mathbf{1 2}}^{+} \cup\left(-\boldsymbol{Q}_{\mathbf{1 2}}^{+}\right): \mathbf{1 2}$ points in $\boldsymbol{C}_{\mathbf{3}}^{*} \quad V_{3} \cup Q_{12}^{+} \cup\left(-Q_{12}^{+}\right)$is a packing set of $r C_{3}^{*}$ for all $0<r \leqslant \frac{3}{5}$.
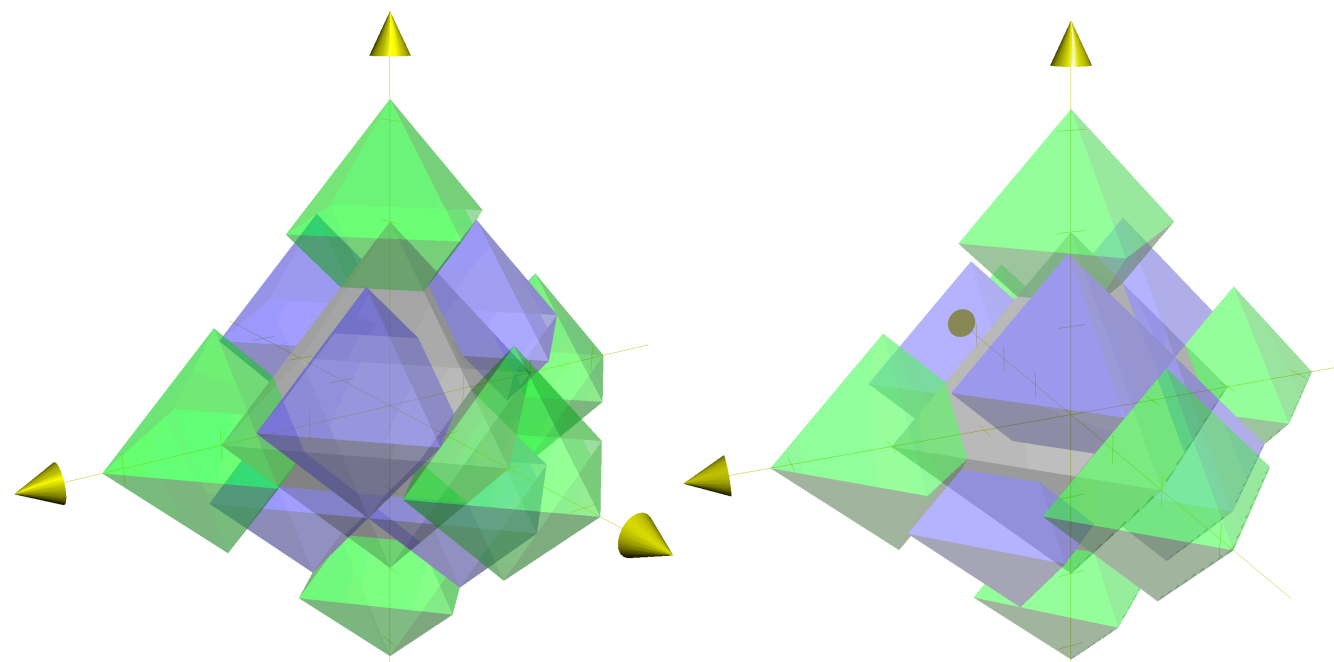

Figure 5: The green cross-polytopes represent the sets $\overline{C\left(\mathbf{x}, \frac{3}{5}\right)}$ for $\mathbf{x} \in V_{3}$ and the blue cross-polytopes represent the sets $\overline{C\left(\mathbf{y}, \frac{3}{5}\right)}$ for $\mathbf{y} \in V_{3} \cup Q_{12}^{+} \cup\left(-Q_{12}^{+}\right)$. 
$V_{3} \cup Q_{13}: 13$ points in $C_{3}^{*} \quad V_{3} \cup Q_{13}$ is a packing set of $r C_{3}^{*}$ for all $0<r \leqslant \frac{6}{11}$.
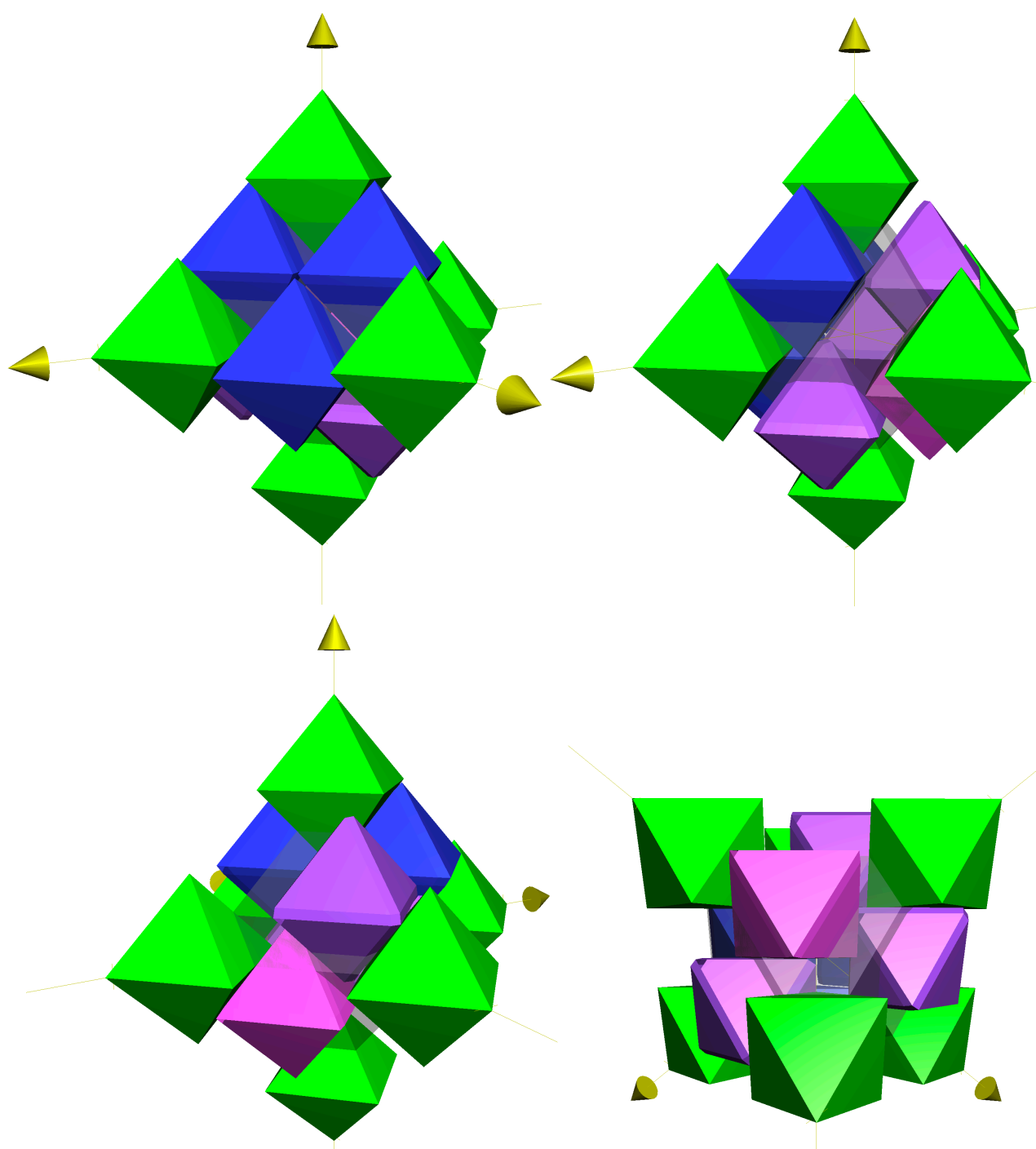

Figure 6: The green cross-polytopes represent the sets $\overline{C\left(\mathrm{x}, \frac{6}{11}\right)}$ for $\mathbf{x} \in V_{3}$, the blue crosspolytopes represent $\overline{C\left(\frac{1}{11}(-1,5,5), \frac{6}{11}\right)}, \overline{C\left(\frac{1}{11}(5,-1,5), \frac{6}{11}\right)}$, and $\overline{C\left(\frac{1}{11}(5,5,-1), \frac{6}{11}\right)}$, the purple cross-polytopes represent $\overline{C\left(\frac{1}{11}(-5,-2,4), \frac{6}{11}\right)}, \overline{C\left(\frac{1}{11}(-5,4,-2), \frac{6}{11}\right)}$, and $\overline{C\left(\frac{1}{11}(4,-2,-5), \frac{6}{11}\right)}$, and the magenta cross-polytope represents $\frac{11}{C\left(\frac{1}{11}(-3,-5,-3), \frac{6}{11}\right)}$.

\section{Acknowledgements}

Thanks to Martin Henk for his advice, suggestions, and feedback, and Fei Xue for feedback and discussions, especially with organizing and simplifying the proof of the $n=3$ and $r \in\left(\frac{3}{5}, \frac{2}{3}\right]$ case. Also I am grateful to the two anonymous reviewers for their comments and suggestions. 


\section{References}

[1] K. Böröczky JR., I. FÁbián, And G. Wintsche, Covering the crosspolytope by equal balls, Periodica Mathematica Hungarica, 53 (2006), pp. 103-113.

[2] J. H. Conway And N. J. A. Slonne, Sphere Packings, Lattices and Groups, Springer-Verlag New York, Inc., 3rd ed., 1998.

[3] H. S. M. Coxeter, Regular Polytopes, Methuen \& Co. Ltd., London, 1948.

[4] H. T. Croft, K. J. Falconer, and R. K. Guy, Unsolved Problems in Geometry, Springer Science+Business Media New York, 1991.

[5] G. Fejes Tóth, F. Fodor, And V. VÍGh, The packing density of the $n$ dimensional cross-polytope, 2015.

[6] L. Fejes Tóth, Lagerungen in der Ebene auf der Kugel und im Raum, SpringerVerlag Berlin Heidelberg, 1953.

[7] L. Fu, W. Steinhardt, H. Zhao, J. E. S. Socolar, and P. Charbonneau, Hard sphere packings within cylinders, Soft Matter, 12 (2016), pp. 2505-2514.

[8] J. E. Goodman, J. O’Rourke, and C. Tóth, Handbook of Discrete and Computational Geometry, Discrete Mathematics and Its Applications, Taylor \& Francis Group, 3rd ed., 2018.

[9] P. M. Gruber, Convex and Discrete Geometry, Springer-Verlag Berlin Heidelberg, 2007.

[10] P. M. Gruber and C. G. Lekkerkerker, Geometry of Numbers, Elsevier Science Publishers B.V., 2nd ed., 1987.

[11] H. HADWIGER, Über treffanzahlen bei translationsgleichen eikörpern, Archiv der Mathematik, 8 (1957), pp. 212-213.

[12] A. B. Hopkins, F. H. Stillinger, and S. Torquato, Densest local spherepacking diversity: General concepts and application to two dimensions, Physical review. E, Statistical, nonlinear, and soft matter physics, 81 (2010), p. 041305.

[13] D. G. LaRman And C. Zong, On the kissing numbers of some special convex bodies, Discrete \& Computational Geometry, 21 (1999), pp. 233-242.

[14] B. D. Lubachevsky And R. L. Graham, Curved hexagonal packings of equal disks in a circle, Discrete \& Computational Geometry, 18 (1997), pp. 179-194.

[15] A. Schürmann, On extremal finite packings, Discrete \& Computational Geometry, 28 (2002), pp. 389-403.

[16] _ _ On packing spheres into containers (about Kepler's finite sphere packing problem), arXiv:math/0506200 (2005).

[17] H. P. F. Swinnerton-Dyer, Extremal lattices of convex bodies, Mathematical Proceedings of the Cambridge Philosophical Society, 49 (1953), pp. 161-162.

[18] I. Talata, On extensive subsets of convex bodies, Periodica Mathematica Hungarica, 38 (1999), pp. 231-246. 
[19] — A lower bound for the translative kissing numbers of simplices, Combinatorica, 20 (2000), pp. 281-293.

[20] G. M. Ziegler, Lectures on Polytopes, Springer Science+Business Media New York, updated seventh printing ed., 2007.

[21] C. Zong, Sphere Packings, Springer-Verlag New York, Inc., 1999. 